\title{
Mechanism of anomalous sinking of an intruder in a granular packing close to incipient fluidization
}

\section{Journal Article}

\section{Author(s):}

Tsuji, Takuya; Penn, Alexander (D); Hattori, Taisuke; Pruessmann, Klaas P.; Müller, Christoph R.; Oshitani, Jun; Washino, Kimiaki; Tanaka, Toshitsugu

Publication date:

2021-06

\section{Permanent link:}

https://doi.org/10.3929/ethz-b-000492883

\section{Rights / license:}

In Copyright - Non-Commercial Use Permitted

\section{Originally published in:}

Physical Review Fluids 6(6), https://doi.org/10.1103/PhysRevFluids.6.064305

\section{Funding acknowledgement:}

182692 - Understanding multi-phase particulate systems: from (reactive) gas-fluidized beds to dense suspensions via advanced magnetic resonance imaging (MRI) and Lagrangian modeling (SNF) 


\title{
Mechanism of anomalous sinking of an intruder in a granular packing close to incipient fluidization
}

\author{
Takuya Tsuji $\odot,{ }^{1, *, \dagger}$ Alexander Penn $\odot, 2,3,4, *, *$ Taisuke Hattori, ${ }^{1}$ Klaas P. Pruessmann, ${ }^{3}$ \\ Christoph R. Müller, ${ }^{2,8}$ Jun Oshitani, ${ }^{5}$ Kimiaki Washino, ${ }^{1}$ and Toshitsugu Tanaka ${ }^{1}$ \\ ${ }^{1}$ Department of Mechanical Engineering, Osaka University, 2-1 Yamadaoka, Suita, Osaka 565-0871, Japan \\ ${ }^{2}$ Laboratory of Energy Science and Engineering, ETH Zürich, Leonhardstrasse 21, 8092 Zürich, Switzerland \\ ${ }^{3}$ Institute for Biomedical Engineering, University of Zürich and ETH Zürich, Gloriastrasse 35, \\ 8092 Zürich, Switzerland \\ ${ }^{4}$ Institute of Process Imaging, Hamburg University of Technology, Denickestraße 17, \\ 21073 Hamburg, Germany \\ ${ }^{5}$ Department of Applied Chemistry and Biotechnology, Okayama University of Science, 1-1 Ridai-cho, \\ Kita-ku, Okayama 700-0005, Japan
}

(Received 19 December 2020; accepted 24 May 2021; published 17 June 2021)

\begin{abstract}
Objects released into a granular packing close to incipient fluidization may float or sink depending on their density. Contrary to intuition, Oshitani et al. [Phys. Rev. Lett. 116, 068001 (2016)] reported that under certain conditions, a lighter sphere can sink further and slower than a heavier one. While this phenomenon has been attributed to a local fluidization around the sinking sphere, its physical mechanisms have not yet been understood. Here, we studied this intriguing phenomenon using both magnetic resonance imaging and discrete particle simulation. Our findings suggest that local fluidization around the sinking sphere and the formation and detachment of gas bubbles play a critical role in driving this anomaly. An analysis of forces acting on the intruder revealed that the upward-directed fluid force acting on a sphere is almost fully counterbalanced by the sum of the net contact forces and the gravitational force acting downward, when the sphere density is close to the bulk density of the granular packing $\left(\rho_{\text {sphere }} / \rho_{\text {bulk }} \approx 1\right)$. At the time when bubbles detach from the sphere, the gas pressure gradient experienced by the sphere is slightly attenuated and the sphere is pushed downward by the particle cap located on top of the sphere. Because the deviations from the force equilibrium are small, the sphere sinks slowly. Even after the sphere has reached its final stable depth, local fluidization in combination with bubble formation remains in the proximity of the sphere.
\end{abstract}

DOI: 10.1103/PhysRevFluids.6.064305

\section{INTRODUCTION}

Granular materials, which are collections of disordered solid particles, are ubiquitous in our daily lives and industrial processes. These materials have attracted considerable attention among physicists and engineers because they sometimes behave similarly to solids, liquids, or gases [1,2] and exhibit diverse and complex mechanical responses to external inputs [3-6]. In many industrial and geophysical processes, the influence of interstitial fluids is important (e.g., on vibration $[7,8]$,

\footnotetext{
*These authors contributed equally to the work.

†tak@mech.eng.osaka-u.ac.jp

†alexander.penn@tuhh.de

${ }^{\S}$ muelchri@ethz.ch
} 


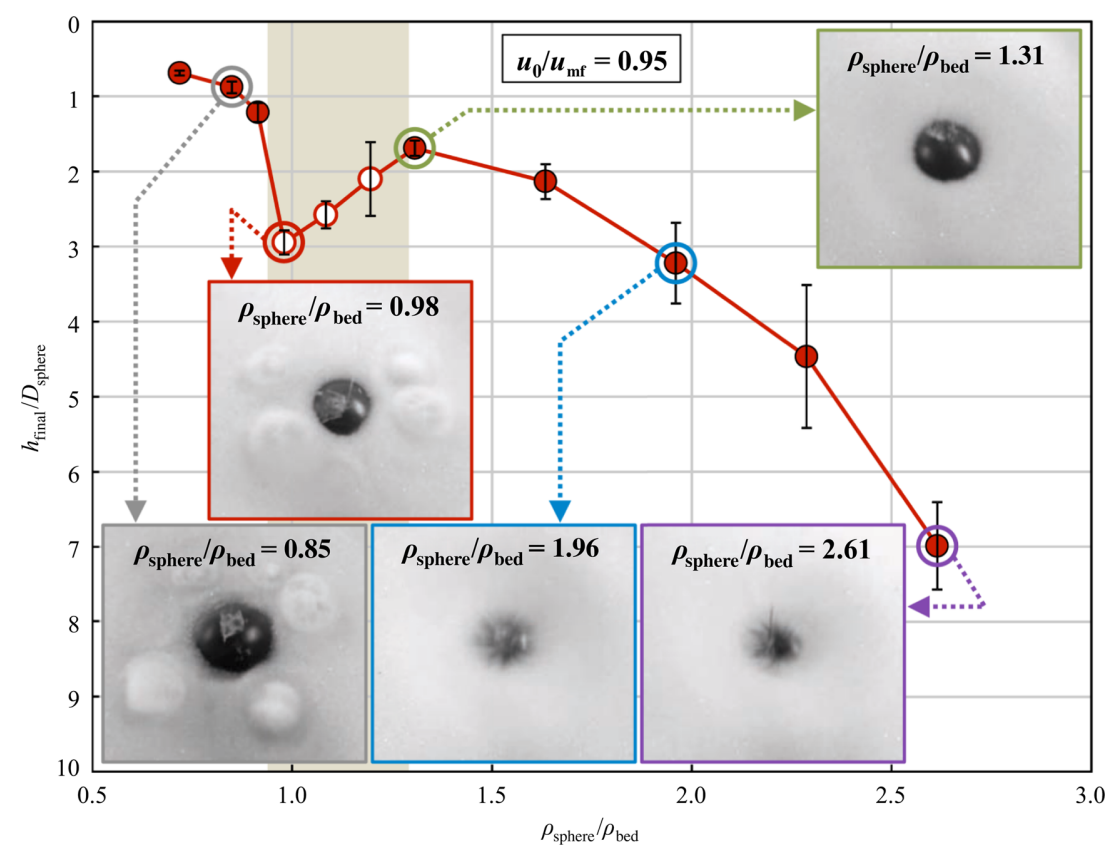

FIG. 1. Final sinking depth of intruding spheres in a powder bed with forced aeration $\left(U_{0} / U_{\mathrm{mf}}=0.95\right.$, $\left.D_{\text {sphere }}=30 \mathrm{~mm}\right)$. Anomalous sphere sinking occurs when the sphere density $\left(\rho_{\text {sphere }}\right)$ is close to the bulk density of a particle bed $\left(\rho_{\text {bed }}\right)$. The final sinking depth $\left(h_{\text {final }}\right)$ is normalized by the sphere diameter $\left(D_{\text {sphere }}\right)$. Obtained from Oshitani et al. [20].

segregation [9-12], and impact [13,14]). Hydrodynamic interactions between granular and fluid phases induce interesting dynamics, such as the emergence of fluidization, and formation of density waves or bubbles [5,15-19].

Oshitani et al. [20] performed a sphere sinking experiment in a powder bed with forced air injection from the bottom. By increasing the flow rate of air, characterized by the superficial velocity $U_{0}$, the bed reaches a state at which the fluid force acting on the particles is balanced by gravity. The fluid velocity at equilibrium is called the minimum fluidization velocity $U_{\mathrm{mf}}$ [15,21]. Above $U_{\mathrm{mf}}$, particles are not in contact with surrounding particles and can easily move. In this fully fluidized state, particles easily mix, and heat and mass transfer between and within phases are enhanced, making this state very attractive for a number of industrial applications, in particular fluidized beds [15,21]. Consequently, a large number of studies have been conducted to characterize the fully fluidized regime $\left(U_{0}>U_{\mathrm{mf}}\right)$. Oshitani et al. [20], in contrast, focused on the regime in which the gas velocity is slightly below $U_{\mathrm{mf}}$. Because the mass of particles is not fully supported by the fluid force in this regime, a bed of granular material is expected to behave like a fixed bed or a porous medium, even though a gas flow exists. They measured the sinking speed and the final sinking depth of the intruding spheres using thin strings attached to the spheres. The experiments were performed by varying the density of the spheres $\left(\rho_{\text {sphere }}\right)$ with respect to the bulk density of the bed $\rho_{\text {bulk }}$ at $U_{0} / U_{\mathrm{mf}}=0.83,0.91$, and $0.95\left(U_{0} / U_{\mathrm{mf}}=1.00\right.$ was also set for comparison). It was shown that the final sinking depth is nonmonotonic with respect to $\rho_{\text {sphere }}$ and that, unexpectedly, spheres sink deeper into a bed when $\rho_{\text {sphere }} / \rho_{\text {bulk }} \approx 1.0$, as shown in Fig. 1 . Furthermore, for this density ratio, the sphere sinking proceeded very slowly and it takes almost 100 times longer to reach their final depth compared to other density cases. These findings are not only of academic curiosity but can guide the development of novel particle separation techniques [22]. Visual observations of the bed surface indicated that local fluidization occurred with bubbles forming around the slowly, deeply sinking 
spheres (Fig. 1). Oshitani et al. [20] argued that local fluidization is responsible for the anomalous sinking; however, the detailed mechanisms of this phenomenon remain poorly understood.

The findings of Oshitani et al. [20] are also interesting from the point of view of the state change of granular materials. $U_{0}=U_{\mathrm{mf}}$ is regarded as a transition point between a contact-dominant solidlike state and a collision-dominant gaslike state. The mechanical response of granular materials to intruders near the transition point is unstable, i.e., the sinking behavior at that point may change drastically in response to small parameter changes. Furthermore, as demonstrated by the formation of bubbles in proximity to the sinking spheres in Fig. 1, a state change can occur locally and intermittently. This means that different states of granular materials can coexist near $U_{\mathrm{mf}}$. As a result, the mechanical responses of granular materials near $U_{\mathrm{mf}}$ might not be explained adequately from macroscopic considerations. Therefore, to understand the anomalous sinking phenomenon, we need to know the spatiotemporal response of a granular material during sinking. However, due to the intrinsic opaqueness and multiscale characteristics of dense granular materials, a detailed experimental observation of their local dynamics is difficult.

Importantly, invasive measurement techniques can disturb the local gas and granular flows and may hence lead to erroneous conclusions. In the past half century, noninvasive imaging techniques for granular systems, such as x-ray radiography [23], x-ray computer tomography [24], electrical capacitance tomography [25], and magnetic resonance imaging (MRI) [26], have been developed.

MRI has the advantage of allowing the nonintrusive, spatially resolved observation of granular flows in any slice of interest. However, MRI suffered from a relatively low temporal resolution, limiting its application to stationary or periodic flow phenomena [26]. Recently, Penn et al. [27] enabled the fast image acquisition of granular flows by a combination of custom-built MR detector arrays, time-efficient single-shot echo-planar imaging sequence, and the engineering of MR-detectable spherical particles. This combination of techniques allows for the fast measurement of granular flows at a temporal resolution of $7 \mathrm{~ms}$ at a spatial resolution of $3 \mathrm{~mm} \times 5 \mathrm{~mm} \times 10$ $\mathrm{mm}$ for a field of view of $200 \mathrm{~mm} \times 300 \mathrm{~mm}$ [27]. Penn et al. successfully observed the dynamic behavior of bubbles in a free-bubbling fluidized bed [27-29]. The same technique was also used to investigate the effects of the addition of small amounts of liquids onto the fluidization characteristics of particles [30], as well as the flow of particles around internal objects in a fluidized bed [31].

In this work, we combined MRI with discrete particle simulation to study why lighter intruding spheres can sink slower and deeper in a bed of aerated particles when compared to heavier spheres. The MRI measurement allowed us to understand how local fluidization and gas bubbling affects the sinking process. The discrete particle simulation, considering the interactions between the sinking spheres, the bed particles, and the gas flows, provided detailed information about the forces acting on the spheres. The combination of both methods allowed for a comprehensive analysis of the sinking anomaly.

\section{METHODS}

\section{A. Setup}

A cylindrical bed with an inner diameter $D$ of $190 \mathrm{~mm}$ and a height $H$ of $300 \mathrm{~mm}$ was constructed from polymethyl methacrylate (PMMA). The distributor was a PMMA plate with 6416 uniformly distributed laser-cut holes with a diameter of $0.5 \mathrm{~mm}$. The distributor had a pressure drop that was at least 10 times larger than that of the particle bed and a homogeneous gas inflow was confirmed [27,32]. Engineered spherical particles consisting of a middle-chain triglyceride oil core and a solid agar shell of diameter $d_{\mathrm{p}}=1.02 \pm 0.12 \mathrm{~mm}$ [27] were used as the bed material. Hollow plastic spheres with a diameter $d_{s}=40 \mathrm{~mm}$ were used as intruders. The spheres were filled with the engineered particles, glass beads, or these mixtures to obtain sphere densities $\rho_{\text {sphere }}$ that are 0.65 , $1.00,1.20$, and 2.50 times larger than the bulk density of the bed material $\rho_{\text {bulk }}=612 \mathrm{~kg} / \mathrm{m}^{3}$. The fluidizing gas was air under ambient condition.

As in the previous study [20], experiments were performed at a superficial inlet gas velocity that is slightly below the minimum fluidization velocity, i.e., $U_{0} / U_{\mathrm{mf}}=0.85-0.97$. Because the 
experiments were performed near equilibrium, the precise measurement of $U_{\mathrm{mf}}$ was important in this study. We fluidized the bed vigorously, achieving $U_{0}>U_{\mathrm{mf}}$, and then gradually defluidized the bed by decreasing the gas inlet velocity carefully, using a programmable mass flow controller (F-203AV, Bronkhorst High-Tech B.V., Netherlands). In a well fluidized state, the gravitational force acting on the total mass of particles is counterbalanced by the fluid force. Thus, we defined $U_{\mathrm{mf}}$ at the intersection between the pressure drop corresponding to the total mass of particles in the bed $\Delta p=W_{\mathrm{p}} g / A$, where $W_{\mathrm{p}}$ and $A$ are the total mass of the particles in the bed and the cross-sectional area of the bed, respectively, and a linear extrapolation of the unfluidized region [32].

To enhance the reproducibility of the experiments, the fluidization and defluidization procedure was performed before each sphere sinking measurement. The initial bed height $H_{0}$ was set to $160 \mathrm{~mm}$. Prior to each measurement, the intruding spheres were centered above the bed, barely touching the bed's surface, using a tube connected to a vacuum pump via a hose. The release of the spheres with no initial velocity was synchronized with the start of the MRI acquisition.

\section{B. Magnetic resonance imaging}

To observe the dynamics of the sinking spheres and the local bubble development near the surface of the spheres, we have employed the real-time MRI measurement as reported by Penn et al. [27]. All MRI experiments were performed on a $3 \mathrm{~T}$ medical MRI scanner (Philips Healthcare, Netherlands). A custom-built 16-channel signal detector array was used for data acquisition. The MR data were reconstructed into image time series using the RECONFRAME software package (Gyrotools LLC, Switzerland).

MRI-based sphere tracking and gas bubbling analysis. A gradient echo MRI sequence was used to measure the local solids volume fraction of the particle phase in the bed. These images were used to track the position of the sinking spheres and to study local gas bubbling. Images were recorded at a temporal resolution of $43 \mathrm{~ms}$ between consecutive frames and a spatial resolution of $2.5 \mathrm{~mm} \times$ $2.5 \mathrm{~mm}$ (horizontal $\times$ vertical) on a central vertical plane through the bed of $10 \mathrm{~mm}$ thickness. The series of images were analyzed using an in-house sphere tracking algorithm based on a twodimensional (2D) cross-correlation of each frame with the reference image of the sphere [32]. Timedependent sphere positions were determined quantitatively by following the maximum of the crosscorrelation. The experiments were repeated at least 3 times for each combination of parameters.

Fluidization-sensitive MRI. In addition to the measurement of the local solid volume fraction, MRI can produce a variety of imaging contrasts. Penn et al. [27] have reported a fluidizationsensitive MRI sequence, which allows one to distinguish between fluidized and jammed regions in a granular packing. In a fluidized state, particles are expected to exhibit incoherent random motion because they are not in contact with surrounding particles. This random motion causes an attenuation of the MRI signal due to the dephasing of excited spins when exposed to a bipolar magnetic field gradient (in this case, the prephaser and the first half of the readout gradient). As a result, fluidized areas exhibit a lower signal intensity (darker contrast) than unfluidized areas. Fluidization-sensitive MRI has been applied successfully to observe the granular response during intruder impact without forced gas injection [27] and local fluidization around an insert in a fluidized bed [31]. The parameters considered in the MRI experiment are listed in Table I.

One-dimensional (1D) MR imaging. The temporal resolution of MRI can be drastically increased if projections onto one single dimension (1D images) are acquired instead of 2D images. Similar to conventional gradient echo MRI, a 2D slice of the sample volume is excited. However, in comparison to the standard scenario, data space is only read along the frequency encoding direction without applying gradient encoding pulses along the phase direction. The resulting 1D images essentially yield projections onto one dimension and were acquired at a temporal resolution of $1.96 \mathrm{~ms}$. Stacked together, these space-time plots allow us to analyze the further detailed motion of the sphere and the gas bubbles. In this work, 1D projections onto the horizontal direction were acquired (averaging over the vertical direction) in order to observe the wiggling motion of the 
TABLE I. Experimental conditions used for the MRI experiments.

\begin{tabular}{lcc}
\hline \hline & Gas (air) & \\
\hline Minimum fluidization velocity: $U_{\mathrm{mf}}(\mathrm{m} / \mathrm{s})$ & & 0.2587 \\
$U_{0} / U_{\mathrm{mf}}$ & Particle & $0.85,0.87,0.89,0.91,0.92,0.93,0.95,0.97$ \\
& & $1.02 \pm 0.12$ \\
\hline Diameter: $d_{\mathrm{p}}(\mathrm{mm})$ & 1036 \\
Density: $\rho_{\mathrm{p}}\left(\mathrm{kg} / \mathrm{m}^{3}\right)$ & & $0.54 \pm 0.05$ \\
Coefficient of friction: $\mu_{\mathrm{p}}$ & Sphere & \\
& & 40 \\
\hline Diameter: $d_{\mathrm{s}}(\mathrm{mm})$ & & $0.65,1.00,1.20,2.50$ \\
$\rho_{\text {sphere }} / \rho_{\text {bulk }}$ & Bed & 190 \\
& & 160 \\
Inner diameter of cylindrical bed: $D(\mathrm{~mm})$ & 0.409 \\
Initial bed height: $H_{0}(\mathrm{~mm})$ & 612 \\
Initial void fraction: $\varepsilon_{0}$ & \\
Bulk density: $\rho_{\text {bulk }}\left(\mathrm{kg} / \mathrm{m}^{3}\right)$ & & \\
\hline \hline
\end{tabular}

sinking spheres. More details on the 1D imaging can be found in [27] where vertical projections have been produced.

\section{Numerical model and parameters}

To numerically investigate local fluidization around sinking spheres on a particle level, a coupled discrete element method (DEM) and computational fluid dynamics (CFD) model [33] was used. Although DEM-CFD has been used widely to examine the interaction between dense particles and gas flows, this method does not allow the consideration of spheres that are significantly larger than the particles in the bed. To address this problem, Tsuji et al. proposed the fictitious particle method (FPM) [34].

Computational cell size $\Delta x$ used for the CFD calculation in the FPM is smaller than the sphere diameter $d_{\mathrm{s}}$ but larger than the particle diameter $d_{\mathrm{p}}$; i.e., $d_{\mathrm{p}}<\Delta x<d_{\mathrm{s}}$. The motion of an incompressible gas flow with density $\rho_{\mathrm{f}}$ and viscosity $\mu_{\mathrm{f}}$ is governed by locally phase-averaged continuity and momentum equations:

$$
\begin{gathered}
\frac{\partial}{\partial t} \varepsilon+\nabla \cdot(\varepsilon \boldsymbol{u})=0, \\
\frac{\partial}{\partial t}(\varepsilon \boldsymbol{u})+\nabla \cdot(\varepsilon \boldsymbol{u} \boldsymbol{u})=-\frac{\varepsilon}{\rho_{\mathrm{f}}} \nabla p+\frac{\varepsilon \mu_{f}}{\rho_{\mathrm{f}}} \nabla^{2} \boldsymbol{u}+\boldsymbol{f},
\end{gathered}
$$

where $\varepsilon, \boldsymbol{u}$, and $p$ are the void fraction in a computational cell, the locally phase-averaged gas velocity, and pressure, respectively. $\boldsymbol{f}$ shows the momentum exchange between gas and solids including particles and spheres:

$$
\boldsymbol{f}=\frac{\beta}{\rho_{\mathrm{f}}}(\overline{\boldsymbol{U}}-\boldsymbol{u})
$$

where $\bar{U}$ is the characteristic velocity of solids existing in a computational cell. $\beta$ is the drag coefficient due to the existence of particles and spheres. Inspired by the volume penalization method (VPM) [35,36], in which a finite-sized solid object is modeled as a porous medium and the object can be regarded as a solid substantially when its permeability becomes sufficiently small, a sphere in the FPM is modeled as an agglomerate of sufficiently small dense fictitious particles when the 


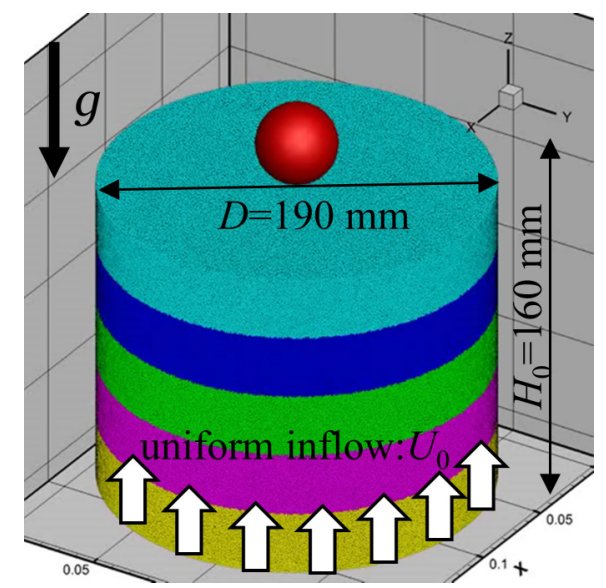

FIG. 2. Computational domain. Particles are colored according to their initial vertical position in the bed.

momentum exchange between the gas and the solids is considered. $\beta$ for a binary mixture of the bed particles and the fictitious particles is obtained by using conventional empirical equations. We used Ergun [37] and Wen and Yu equations [38] depending on the local solid concentration.

Particle and sphere motions are governed by translational and rotational equations of motion individually. For particle $i$,

$$
\begin{gathered}
m_{\mathrm{p}} \dot{\boldsymbol{U}}_{\mathrm{p} i}=\boldsymbol{f}_{\mathrm{Cp} i}+\boldsymbol{f}_{\mathrm{Fp} i}+m_{\mathrm{p}} \boldsymbol{g}, \\
I_{\mathrm{p}} \dot{\boldsymbol{\omega}}_{\mathrm{p} i}=\boldsymbol{M}_{\mathrm{p} i},
\end{gathered}
$$

where $m_{\mathrm{p}}$ and $I_{\mathrm{p}}$ are the mass and moment of inertia of a particle; $\boldsymbol{U}_{\mathrm{p} i}$ and $\boldsymbol{\omega}_{\mathrm{p} i}$ are velocity and angular velocity of a particle $i ; g$ is the gravitational acceleration; $f_{\mathrm{Cp} i}$ is the net contact force working on a particle $i$ from the other particles, spheres, and walls in contact; $f_{\mathrm{Fp} i}$ is the fluid force working on a particle $i$; and $\boldsymbol{M}_{\mathrm{p} i}$ is the net torque working on a particle $i$ due to the contacts with other particles, spheres, and walls. For sphere $j$,

$$
\begin{gathered}
m_{\mathrm{s}} \dot{\boldsymbol{U}}_{\mathrm{s} j}=\boldsymbol{f}_{\mathrm{Cs} j}+\boldsymbol{f}_{\mathrm{Fs} j}+m_{\mathrm{s}} \boldsymbol{g}, \\
I_{\mathrm{s}} \dot{\boldsymbol{\omega}}_{\mathrm{s} j}=\boldsymbol{M}_{\mathrm{s} j},
\end{gathered}
$$

where $m_{s}$ and $I_{s}$ are the mass and moment of inertia of a sphere; $\boldsymbol{U}_{\mathrm{s} j}$ and $\boldsymbol{\omega}_{\mathrm{s} j}$ are velocity and angular velocity of a sphere $j ; f_{\mathrm{Cs} j}$ is the net contact force working on a sphere $j$ from the particles, other spheres, and walls in contact; $f_{\mathrm{Fs} j}$ is the fluid force working on a sphere $j$; and $M_{\mathrm{s} j}$ is the net torque working on sphere $j$ due to the contacts with particles, walls, and fluids. All the contact interactions were described by a DEM model consisting of linear springs, dashpots, and a frictional slider. A rolling resistance model was also included to suppress excessive relative rotations at contact points $[39,40]$. Because the FPM captures gas flows in the scales larger than CFD cell $\left(\Delta x \approx 2-3 d_{\mathrm{p}}\right)$, it enables us to directly observe the formation of local bubbles. More details on the FPM can be found in [34].

The parameters considered in the numerical study are shown in Table II and the dimensions of the cylindrical bed used in the MRI experiment were used for the calculations (Fig. 2). At the bottom of the bed, a uniform air inflow was assumed and the pressure at the top of the bed was kept equal to the atmospheric pressure. To avoid crystallization of particles in the bed, a narrow particle size distribution was considered. We kept the mean particle diameter equal to that in the MRI experiment $\left(\bar{d}_{\mathrm{p}}=1.02 \mathrm{~mm}\right)$ and a mixture of particles with diameters $d_{p}=0.92,1.02$, and $1.12 \mathrm{~mm}$ was used (number ratio of 1:2:1). The density of the spherical intruder $\rho_{\text {sphere }}$ was defined 
TABLE II. Parameters used in the numerical simulation.

\begin{tabular}{|c|c|}
\hline \multicolumn{2}{|c|}{ Gas (air) } \\
\hline $\begin{array}{l}\text { Density: } \rho_{\mathrm{f}}\left(\mathrm{kg} / \mathrm{m}^{3}\right) \\
\text { Viscosity: } \mu_{\mathrm{f}}(\mathrm{Pa} \mathrm{s}) \\
\text { Minimum fluidization velocity: } U_{\mathrm{mf}}(\mathrm{m} / \mathrm{s}) \\
U_{0} / U_{\mathrm{mf}}\end{array}$ & $\begin{array}{c}1.205 \\
1.81 \times 10^{-5} \\
0.3008 \\
0.93,0.935,0.938,0.940,0.95\end{array}$ \\
\hline \multicolumn{2}{|c|}{ Particle } \\
\hline $\begin{array}{l}\text { Diameter: } d_{\mathrm{p}}(\mathrm{mm}) \\
\text { Density: } \rho_{\mathrm{p}}\left(\mathrm{kg} / \mathrm{m}^{3}\right) \\
\text { Number of particles: } N_{\mathrm{p}}\end{array}$ & $\begin{array}{c}0.92,1.02,1.12(1: 2: 1) \\
1036 \\
4743596\end{array}$ \\
\hline $\begin{array}{l}\text { Diameter: } d_{\mathrm{s}}(\mathrm{mm}) \\
\rho_{\text {sphere }} / \rho_{\text {bulk }} \\
\text { Diameter of fictitious particle: } d_{\text {fic }}(\mathrm{mm}) \\
\text { Filling fraction of fictitious particle: } \alpha_{\text {fic }}\end{array}$ & $\begin{array}{c}40 \\
0.65,1.00,1.20,1.40,1.50,1.80,2.00,2.50 \\
0.51 \\
0.74\end{array}$ \\
\hline $\begin{array}{l}\text { Normal spring constant: } k_{\mathrm{n}}(\mathrm{N} / \mathrm{m}) \\
\text { Tangential spring constant: } k_{\mathrm{t}}(\mathrm{N} / \mathrm{m}) \\
\text { Coefficient of restitution: } e_{\mathrm{p}} \\
\text { Coefficient of friction: } \mu_{\mathrm{p}} \\
\text { Coefficient of rolling resistance: } \mu_{\mathrm{r}}\end{array}$ & $\begin{array}{l}800 \\
200 \\
0.90 \\
0.35 \\
0.01\end{array}$ \\
\hline $\begin{array}{l}\text { Inner diameter of cylindrical bed: } D(\mathrm{~mm}) \\
\text { Initial bed height: } H_{0}(\mathrm{~mm}) \\
\text { Initial void fraction: } \varepsilon_{0} \\
\text { Bulk density: } \rho_{\text {bulk }}\left(\mathrm{kg} / \mathrm{m}^{3}\right) \\
\text { Calculation domain: } L_{x} \times L_{y} \times L_{z}(\mathrm{~mm}) \\
\text { Number of CFD cells: } n_{x} \times n_{y} \times n_{z} \\
\text { Time increment: } \Delta t(\mathrm{~s})\end{array}$ & $\begin{aligned} 190 \\
160 \\
0.403 \\
618.5 \\
190 \times 190 \times 275 \\
75 \times 75 \times 109 \\
1.0 \times 10^{-5}\end{aligned}$ \\
\hline
\end{tabular}

relative to the bulk density of the bed $\rho_{\text {bulk }}=618.5 \mathrm{~kg} / \mathrm{m}^{3}$ and set to $\rho_{\text {sphere }} / \rho_{\text {bulk }}=0.65,1.00,1.20$, and 2.50 corresponding to the values of the MRI experiments. In addition, $\rho_{\text {sphere }} / \rho_{\text {bulk }}=1.40,1.50$, 1.80 , and 2.00 were also investigated in the simulation. To simplify the simulations, the same DEM parameters (spring constants, restitution coefficient, coefficient of friction, and coefficient of rolling resistance) were used for all contacts. As the spring constant does not affect the numerical simulations of a fluidized state $\left(U_{0}>U_{\mathrm{mf}}\right)$, a softer spring is commonly used to reduce computational costs $[33,41]$. We checked whether the sinking of a sphere is dependent on the spring constant and found that the sinking speed and final depth of a sphere did not change significantly over a variation of the spring constant by three orders of magnitude (the results are shown in Appendix A). To reduce the computational cost, we used $k_{\mathrm{n}}=800 \mathrm{~N} / \mathrm{m}$ and $k_{\mathrm{t}}=200 \mathrm{~N} / \mathrm{m}$ for the normal and tangential directions, respectively. We also checked the dependency of the sinking dynamics of the intruding sphere on other DEM parameters and found that the friction coefficient affects the sinking behavior; here we used $\mu_{\mathrm{p}}=0.35$ for the simulation which is slightly smaller than the value determined experimentally $\mu_{\mathrm{p}}=0.54 \pm 0.05$ (the results are shown in Appendix B).

Similar to the MRI experiments, we performed a fluidization and defluidization procedure to obtain an initial random loose packing. The coefficient of friction was increased during the fluidization and defluidization processes. After the filling process of the bed and once the particles 
have settled down, the coefficient was reset to the target value [40,42]. The bed was relaxed again until it reached an equilibrium state. The initial void fraction of the bed as obtained by DEM $\left(\varepsilon_{0}=\right.$ $0.403)$ was close to that in the MRI experiments $\left(\varepsilon_{0}=0.409\right)$ and the random loose packing limit of monodisperse particles under gravity $\left(\varepsilon_{\mathrm{RLP}}=0.4\right)$ [43]. $U_{\mathrm{mf}}$ was defined according to the procedure in the experiment. We used a uniform Cartesian grid and approximately 16 computational cells per sphere diameter $\left(d_{\mathrm{s}} / \Delta x \approx 16\right)$ for the CFD calculations. This spatial resolution is sufficiently high to capture the gas pressure field around the spheres, which is an important characteristic to explain the sinking anomaly. All calculations were performed using a fully parallelized in-house code.

\section{RESULTS AND DISCUSSIONS}

\section{A. Sphere sinking with local fluidization at $U_{0} / U_{\mathrm{mf}}=0.95$}

Figure 3(a) shows selected frames of the MR image series that were recorded at $U_{0} / U_{\mathrm{mf}}=0.95$ in a central vertical slice through the bed containing spheres of different densities. The high spatiotemporal resolution allowed us to observe differences in the sinking dynamics as a function of sphere density. Spheres of different densities appear with a different contrast owing to the differences in the MR activity of the materials that were used to fill the spheres. Figure 4(a) plots sinking trajectories as a function of time, as extracted from the MR images displayed in Fig. 3(a). Average values and standard deviations of the time-dependent sphere positions were obtained from the analysis of at least three repetitions for each experiment. The largest standard deviations were observed for $\rho_{\text {sphere }} / \rho_{\text {bulk }}=1.0$; for this case the averaging was performed over seven repetitions to increase the robustness of the analysis. This increased standard deviation indicates that the slow and deep sinking is more susceptible to very small variations in the experimental conditions, such as small fluctuations in the superficial velocity or local variations in packing fraction when compared to the other density ratios.

Figure 3(b) is an MR image of a sphere with $\rho_{\text {sphere }} / \rho_{\text {bulk }}=0.65$ after it reached the steady state. The sphere is partially submerged in the bed and bubbles are formed frequently in the proximity of the sphere. The sphere with $\rho_{\text {sphere }} / \rho_{\text {bulk }}=1.00$ sinks further down the bed and sinks for a longer time than the heavier sphere $\rho_{\text {sphere }} / \rho_{\text {bulk }}=1.20$; the sphere with $\rho_{\text {sphere }} / \rho_{\text {bulk }}=1.00$ took almost $8 \mathrm{~s}$ to reach its final depth, while all of the other spheres reached their final depths within $0.5 \mathrm{~s}$. As demonstrated in Fig. 3(c), during the slow sinking of sphere $\rho_{\text {sphere }} / \rho_{\text {bulk }}=1.00$, bubbles form and detach from the sinking sphere (see Supplemental Video 1 in the Supplemental Material [44]). Figure 5 shows a time series of $1 \mathrm{D}$ MR images of a sinking sphere with $\rho_{\text {sphere }} / \rho_{\text {bulk }}=1.00$. The image is averaged along the vertical direction. The lateral wiggling motion during the slow sinking can be observed. Dark contrasts outside the sphere represent bubbles. The experimental data indicate that the sphere is being attracted to the sides where detached bubbles exist. This is also observed in 2D measurements (see Supplemental Video 2 [44]). The dataset shown in Fig. 5 as well as another dataset produced with the same experimental conditions was used to roughly estimate the bubbling frequency to $2.6 \pm 0.60 \mathrm{~Hz}$. Although multiple bubbles can coexist at a time as demonstrated in Fig. 1, this analysis only detects bubbles that occur within the observed field of view, which is a slice of thickness $10 \mathrm{~mm}$. Bubbles that are produced outside this field of view are not included in this analysis, nor is horizontal through-plane motion of the sphere visible. Hence the frequency reported here is only a lower bound to the real frequency and should only serve as a rough estimate.

Figure 6 shows fluidization-sensitive MRI images of the intruding sphere with $\rho_{\text {sphere }} / \rho_{\text {bulk }}=$ 1.00 (see Supplemental Video 3 [44]). The measurement was performed with a temporal resolution of $546 \mathrm{~ms}$ and a spatial resolution $2.5 \mathrm{~mm} \times 2.5 \mathrm{~mm} \times 10.0 \mathrm{~mm}$. Because the particles are static before the release of the spheres, a uniform signal intensity can be observed throughout the bed [Fig. 6(a)]. Immediately after the impact of the sphere, an inverted cone-shaped region, with a darker contrast, appeared from the bottom of the sphere up to the bed surface [Fig. 6(b)]. The potential energy of the sphere is transferred to the particles in this region, and local fluidization might occur. The frequent inelastic collisions between particles in the region lead to rapid energy 


\section{(a)}
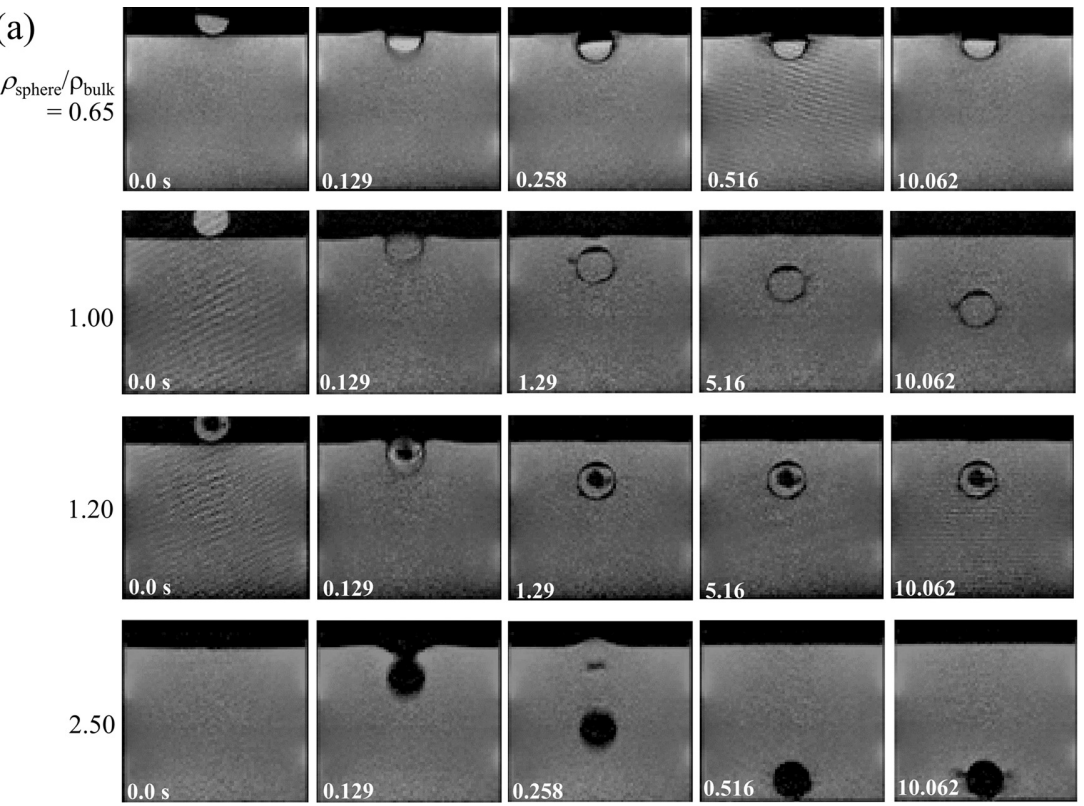

(b)

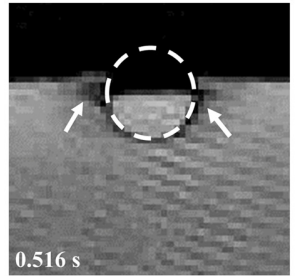

(c)
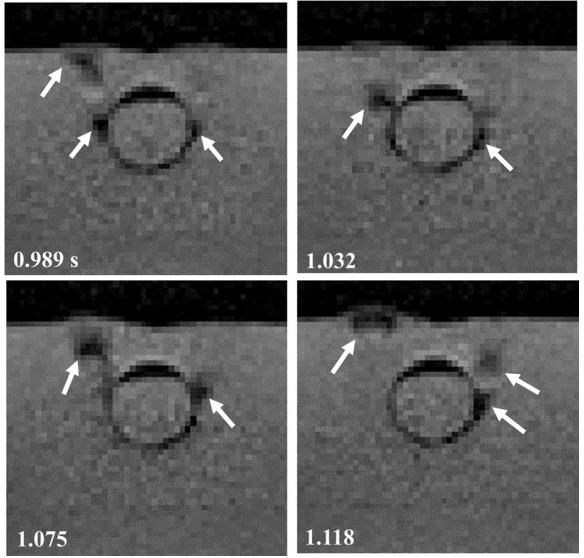

FIG. 3. (a) MR images of spheres of different densities (rows) sinking into a bed that is kept at near incipient fluidization $\left(U_{0} / U_{\mathrm{mf}}=0.95\right)$. Note that the time increments vary with $\rho_{\text {sphere }} / \rho_{\text {bulk }}$. (b) Bubble formation near a partially submerged sphere $\left(\rho_{\text {sphere }} / \rho_{\text {bulk }}=0.65\right)$. Dashed line and arrows indicate the perimeter of the sphere and bubbles, respectively. (c) Bubble formation in the proximity of a slow sinking sphere $\left(\rho_{\text {sphere }} / \rho_{\text {bulk }}=1.00\right)$. The dynamics of the detaching bubbles are clearly observable (see Supplemental Video $1[44])$.

dissipation and to a fast reduction of the local fluidization region. During the slow and deep sinking of sphere $\rho_{\text {sphere }} / \rho_{\text {bulk }}=1.00$ [Fig. 6(c)], the fluidized region is limited to the area surrounding the sinking sphere up to the bed surface; in this region, the bubbles that have formed at the sphere pass through. Figure 7 shows time-averaged fluidization-sensitive MRI images after the spheres have reached their final depths. It is worth mentioning that, when the bubble detachment stopped, the fluidized region above the sphere quickly shrank, and the sphere did not sink any further into the packing. After the sinking motion of the intruder spheres had stopped, however, the local fluidization and bubbling below and around the sphere remained. Unlike the spheres with $\rho_{\text {sphere }} / \rho_{\text {bulk }}=0.65$ 


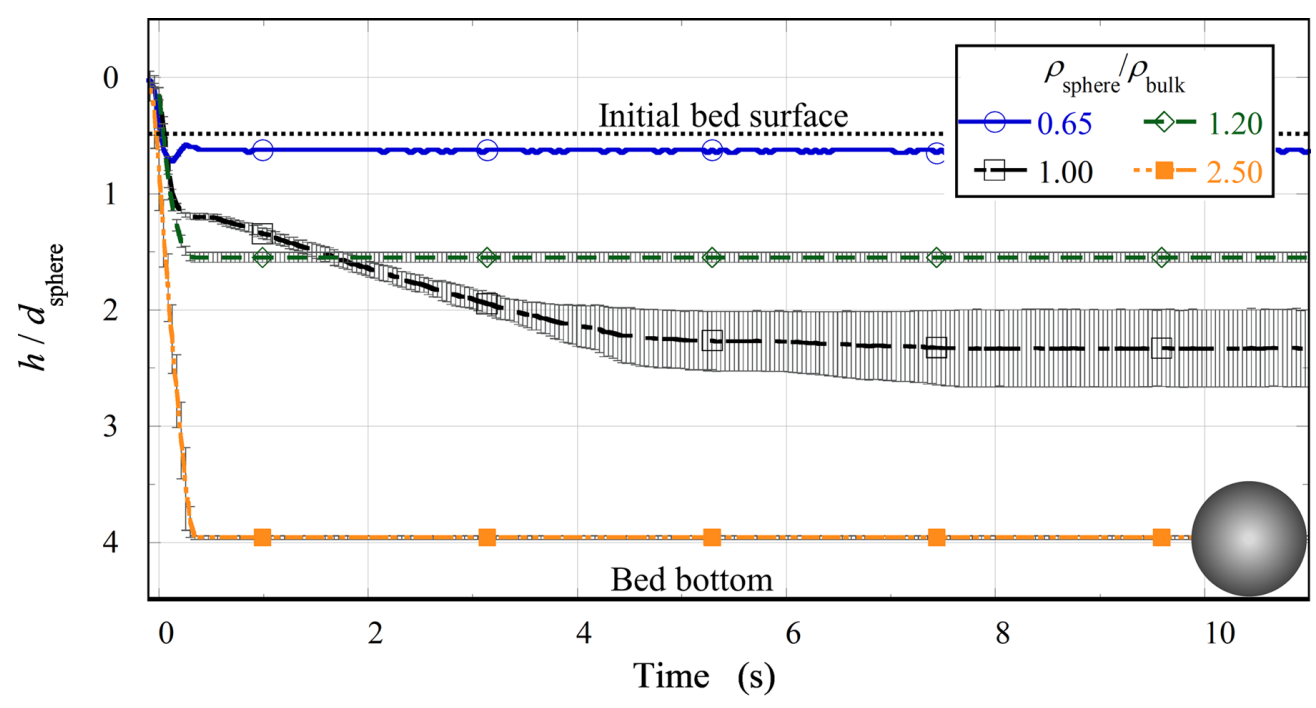

(a) MRI experiments

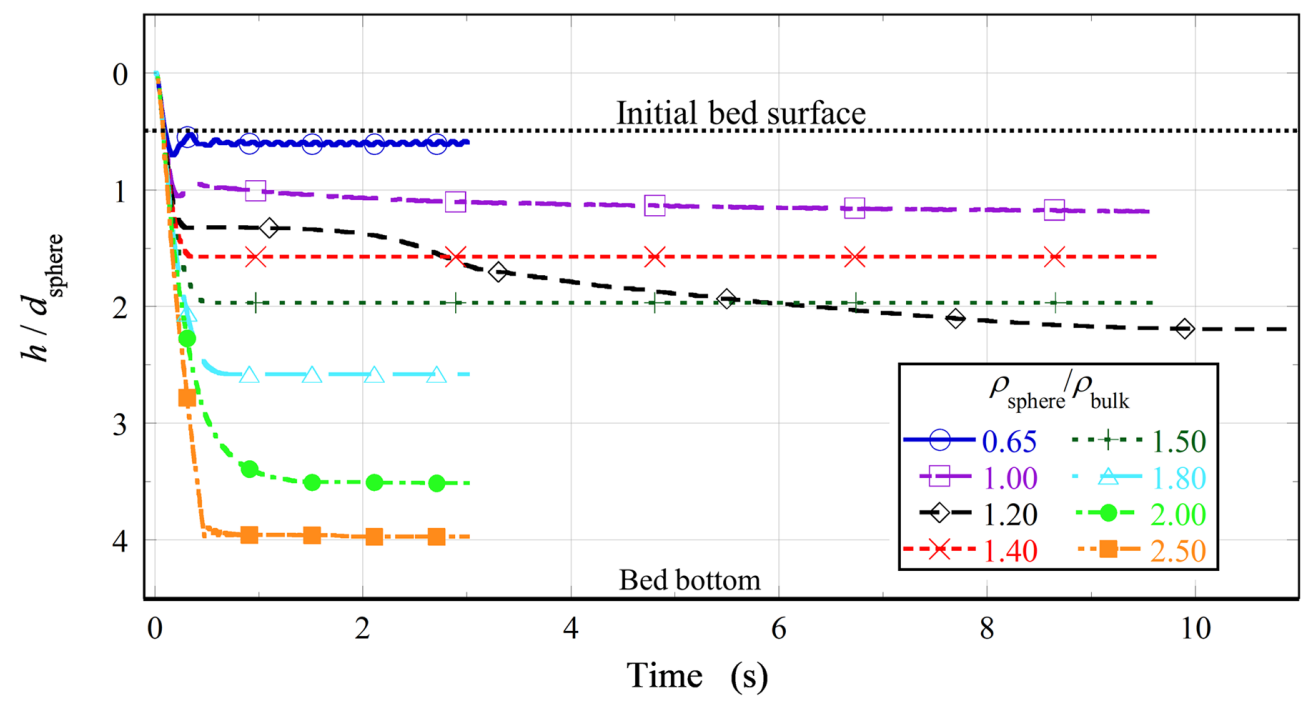

(b) Numerical simulations

FIG. 4. Sphere sinking trajectories at $U_{0} / U_{\mathrm{mf}}=0.95$. $h$ is the center position of the spheres and increases downward. The initial bed surface was located at $h / d_{\text {sphere }}=0.5$. The heaviest sphere $\rho_{\text {sphere }} / \rho_{\text {bulk }}=2.50$ reached the bottom inlet of the bed in both the MRI experiment and the simulation.

and 1.00, we could observe neither bubble formation nor bubble detachment for the sphere with $\rho_{\text {sphere }} / \rho_{\text {bulk }}=1.20$ both during and after sinking; this might be caused by an increased force chain network underneath the spheres that supports the weight of the sphere and prevents local fluidization. We observed some bubble formation for the heaviest sphere with $\rho_{\text {sphere }} / \rho_{\text {bulk }}=2.50$, but exclude this observation from our discussion of the sinking and stopping mechanisms because this sphere reached the bottom of the bed and was supported by the bottom gas inlet.

Turning now to our numerical results, Figure 8(a) shows contour plots of the local void fraction measured at each particle center $\varepsilon_{\text {local }}$ [40,45] (see Supplemental Video 4 [44]). All of 

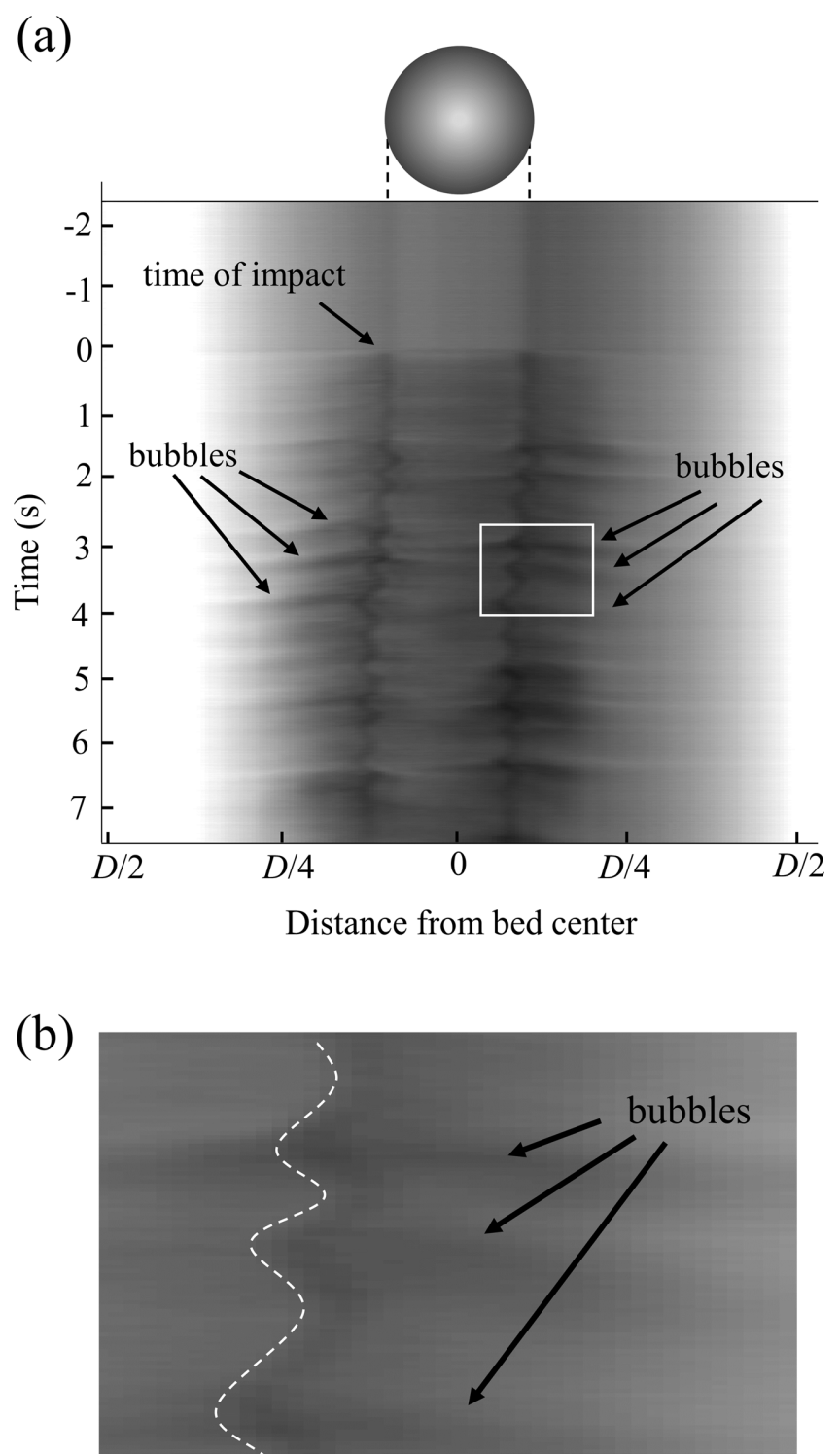

FIG. 5. (a) A time series of 1D horizontal MR images of a sinking sphere with $\rho_{\text {sphere }} / \rho_{\text {bulk }}=1.00$. Dark contrasts outside the sphere represent bubbles. This measurement was performed at a temporal resolution of 1.96 ms. (b) Magnified view. Dashed line shows the sphere surface. Contrasts in these figures are adjusted to enhance visibility.

the simulations were initialized with the same particle packing. We have put great effort into achieving a uniform random packing, but a nonuniformity in the local void fraction in the range of $\varepsilon_{\text {local }}=0.370-0.452$ remained. Figures $8(\mathrm{~b})$ and $8(\mathrm{c})$ show the three-dimensional void structures that formed inside the bed for $\rho_{\text {sphere }} / \rho_{\text {bulk }}=0.65$ and 1.20 , respectively (see Supplemental Video 5 [44]). Similar to our MRI results, we observed local bubbling for the lighter spheres $\left(\rho_{\text {sphere }} / \rho_{\text {bulk }}=\right.$ $0.65,1.00$, and 1.20$)$ but not for the heavier spheres $\left(\rho_{\text {sphere }} / \rho_{\text {bulk }}=1.40,1.50,1.80\right.$, and 2.00$)$. The heaviest sphere $\left(\rho_{\text {sphere }} / \rho_{\text {bulk }}=2.50\right)$ reached the bottom of the bed.

Figure 4(b) plots the numerically determined trajectories of the sinking spheres. Because the spheres reached the final stable depths and no further sinking was observed, the calculations were 


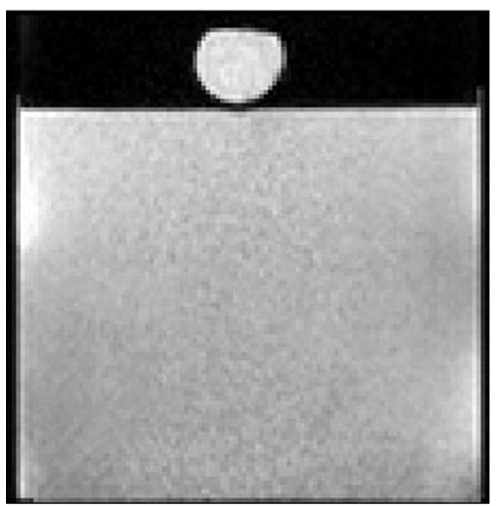

(a) Before release

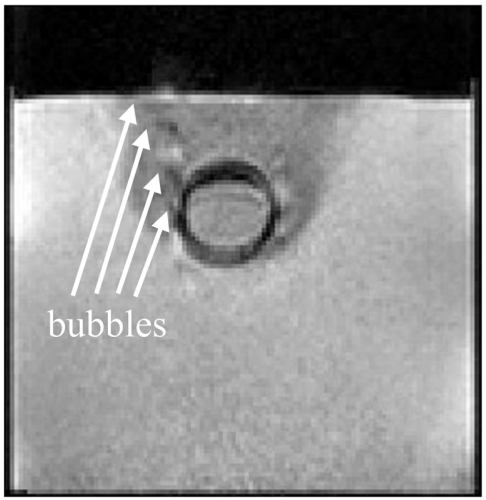

(c) During slow and deep sinking

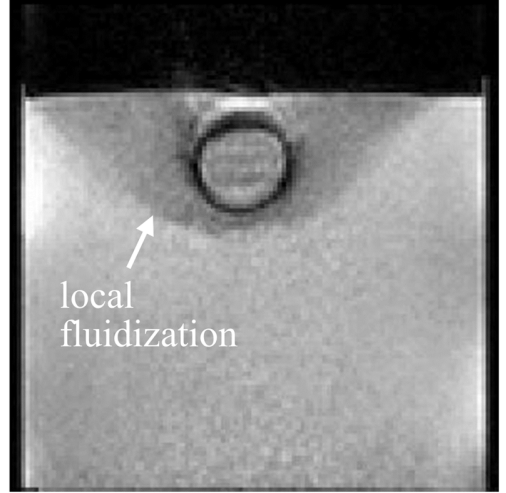

(b) After impact

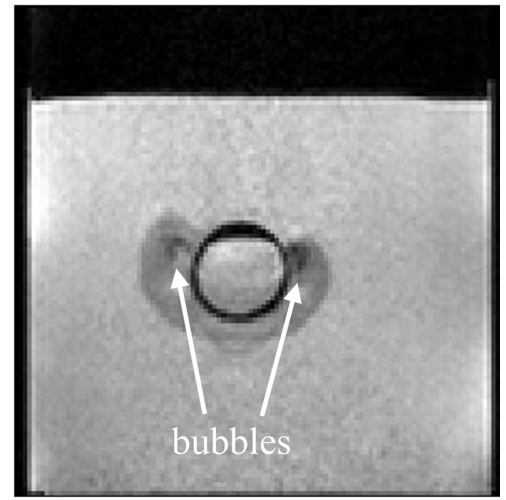

(d) After stop

FIG. 6. Fluidization-sensitive MR images $\left(\rho_{\text {sphere }} / \rho_{\text {bulk }}=1.00, U_{0} / U_{\mathrm{mf}}=0.95\right)$. During the slow and deep sinking, the sphere is surrounded by fluidized granular material (darker contrast) while gas bubbles detach from the sides of the sphere and reach the free surface. Once this chain of bubbles stops (i.e., bubbles do not reach the top surface of the bed anymore), the cap region above the sphere defluidizes (higher contrast) and the sphere stops sinking. A fluidized region (darker contrast) with some bubble formation remains at the sides and below the sphere. The relatively long acquisition time of $546 \mathrm{~ms}$ creates motion blurring for the sinking sphere (see Supplemental Video 3 [44]).

terminated after $3.0 \mathrm{~s}$ for $\rho_{\text {sphere }} / \rho_{\text {bulk }}=0.65,1.80,2.00$, and 2.50 and after $9.5 \mathrm{~s}$ for $\rho_{\text {sphere }} / \rho_{\text {bulk }}=$ $1.00,1.40$, and 1.50 . We observed that the sphere $\rho_{\text {sphere }} / \rho_{\text {bulk }}=1.20$ sank to a deeper position than the heavier spheres $\rho_{\text {sphere }} / \rho_{\text {bulk }}=1.40$ and 1.50. Figure 9 compares the final sinking depths obtained from the MRI experiments and the numerical simulations. Both the experiments and the simulations reproduced the anomalous sinking reported previously [20]; i.e., a slow and deep sinking occurred when the sphere density was similar to the bulk density of the bed $\left(\rho_{\text {sphere }} \approx \rho_{\text {bulk }}\right)$. However, some small differences exist between the MRI experiments and the numerical results. In particular, the slow and deep sinking occurred for a slightly heavier sphere in the numerical simulations $\left(\rho_{\text {sphere }} / \rho_{\text {bulk }}=1.20\right)$ compared to the MRI experiments $\left(\rho_{\text {sphere }} / \rho_{\text {bulk }}=1.00\right)$. In Fig. 9 , linear least-squares approximations excluding the anomalous results $\left(\rho_{\text {sphere }} / \rho_{\text {bulk }}=1.00\right.$ in the MRI experiment and 1.20 in the simulation) are also plotted. The final sinking depths increased linearly with the sphere density except in the anomalous cases. This result is similar to the sinking 


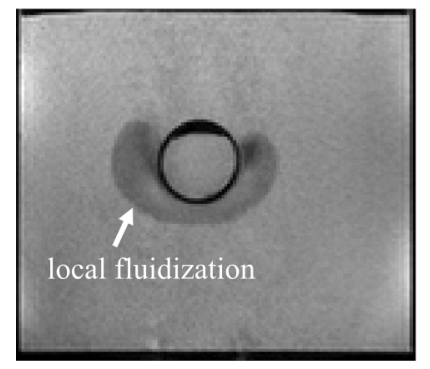

$\rho_{\text {sphere }} / \rho_{\text {bulk }}=1.00$

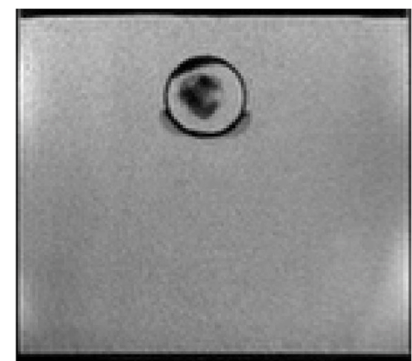

1.20

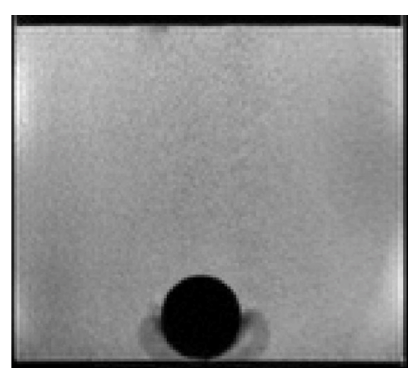

2.50

FIG. 7. Time-averaged fluidization-sensitive MR images for intruding spheres with $\rho_{\text {sphere }} / \rho_{\text {bulk }}=1.00$, 1.20 , and 2.50 after reaching their final depths $\left(U_{0} / U_{\mathrm{mf}}=0.95\right)$.

of an intruder in a very loosely packed bed of fine sand $\left(\varepsilon_{0}=0.59\right)$ in the absence of a forced gas flow [46].

It should be noted that the engineered particles used in this study had a diameter that is almost 4 times larger and a 2.5 times smaller particle density than the glass beads used previously [20], indicating that the observed anomaly occurs across a range of particle sizes and densities. In the following, the mechanisms of the sinking and stopping are elucidated by consolidating the insights gained experimentally and numerically.

\section{B. Sinking and stopping mechanisms as a function of the density ratio $\rho_{\text {sphere }} / \rho_{\text {bulk }}$}

The sinking behaviors observed in the previous section can be categorized depending on $\rho_{\text {sphere }} / \rho_{\text {bulk }}$ : (i) floating near the bed surface $\left(\rho_{\text {sphere }} / \rho_{\text {bulk }}=0.65\right.$ in the MRI experiment and $\rho_{\text {sphere }} / \rho_{\text {bulk }}=0.65$ and 1.00 in the simulation); (ii) anomalous slow and deep sinking ( $\rho_{\text {sphere }} / \rho_{\text {bulk }}=1.00$ in the MRI experiment and $\rho_{\text {sphere }} / \rho_{\text {bulk }}=1.20$ in the simulation), and (iii) rapid sinking $\left(\rho_{\text {sphere }} / \rho_{\text {bulk }}=1.20\right.$ and 2.50 in the MRI experiment and $\rho_{\text {sphere }} / \rho_{\text {bulk }}=1.40,1.50$, $1.80,2.00$, and 2.50 in the simulation). In the following subsections, these are described in detail and the underlying mechanisms are discussed.

\section{Floating of light spheres $\left(\rho_{\text {sphere }} / \rho_{\text {bulk }}<1\right)$}

Figure 10 plots the vertical forces acting on a sphere with $\rho_{\text {sphere }} / \rho_{\text {bulk }}=0.65$ as determined by discrete particle simulations. The total force is the sum of the fluid, net contact, and gravitational forces. All forces were normalized with respect to gravity, and positive forces act vertically upward.

During the initial period of sphere impact $(t \leqslant 0.16 \mathrm{~s})$, a rapid increase in the upward-directed contact force was observed. Subsequently, the magnitude of the fluid force increased, with some fluctuations, and reached a steady state at $t \approx 0.2 \mathrm{~s}$. The experimental [Fig. 3(b)] and numerical [Fig. 8(b)] results indicate that the floating sphere is accompanied by frequent bubble formations from the bottom of the sphere; the detached bubbles move along the sides of the sphere. The fluctuations in the fluid force, and the total force, are related to sequential bubble formation and detachment events. Once a steady state is reached $(t>0.2 \mathrm{~s})$, the contribution of the contact force becomes small, and the gravitational force is almost fully counterbalanced by the fluid force. As schematically shown in Fig. 11, when a gas is continuously injected into a uniformly packed, granular bed under gravity, a gas pressure drop occurs in the flow direction due to the fluid drag force acting on the particles. Hence, a sphere that is placed within the bed will experience a vertical, upward-directed force due to the negative gas pressure gradient, similar to the Archimedean buoyant force (with the difference that the Archimedean buoyant force is static and does not necessarily require a flow). Because the working fluid is air and the frictional drag force directly acting on the sphere surface is negligible, the buoyant and form drag forces, both arising from the gas pressure 
(a)
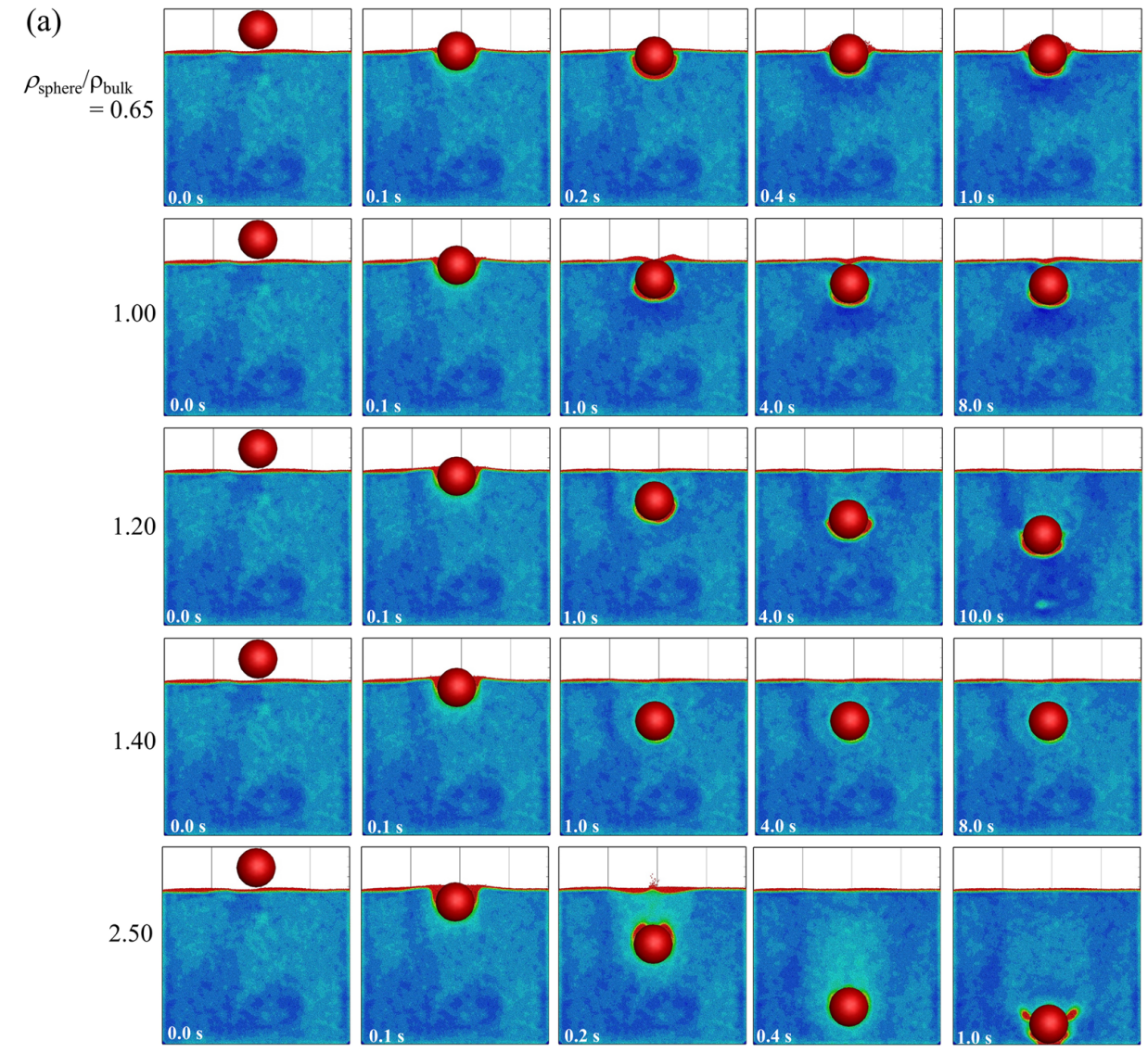

(b)
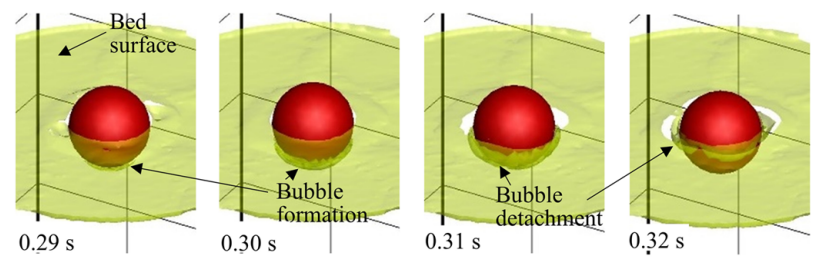

(c)
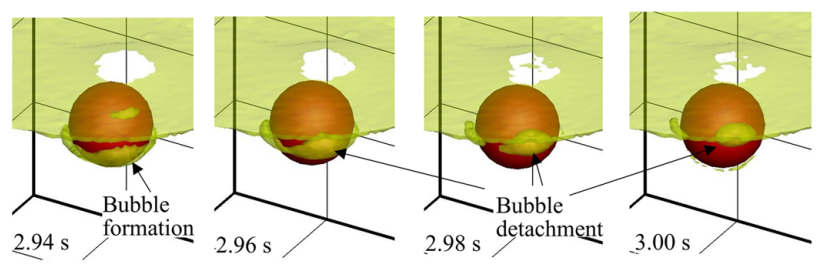

FIG. 8. (a) Numerical results of spheres of different densities (rows) sinking into a bed that is kept near incipient fluidization $\left(U_{0} / U_{\mathrm{mf}}=0.95\right)$. The contours reflect the local void fraction. The results for the density ratios $\rho_{\text {sphere }} / \rho_{\text {bulk }}=0.65,1.00,1.20,1.40$, and 2.50 are shown. The time increments depend on $\rho_{\text {sphere }} / \rho_{\text {bulk }}$ (see Supplemental Video 4 [44]). (b) Three-dimensional presentation of the void structures inside the bed $\left(\rho_{\text {sphere }} / \rho_{\text {bulk }}=0.65\right)$. The isosurface of the local void fraction $\varepsilon_{\text {local }}=0.80$ is plotted. (c) Three-dimensional presentation of the void structures inside the bed $\left(\rho_{\text {sphere }} / \rho_{\text {bulk }}=1.20\right)$ (see Supplemental Video 5 [44]). 


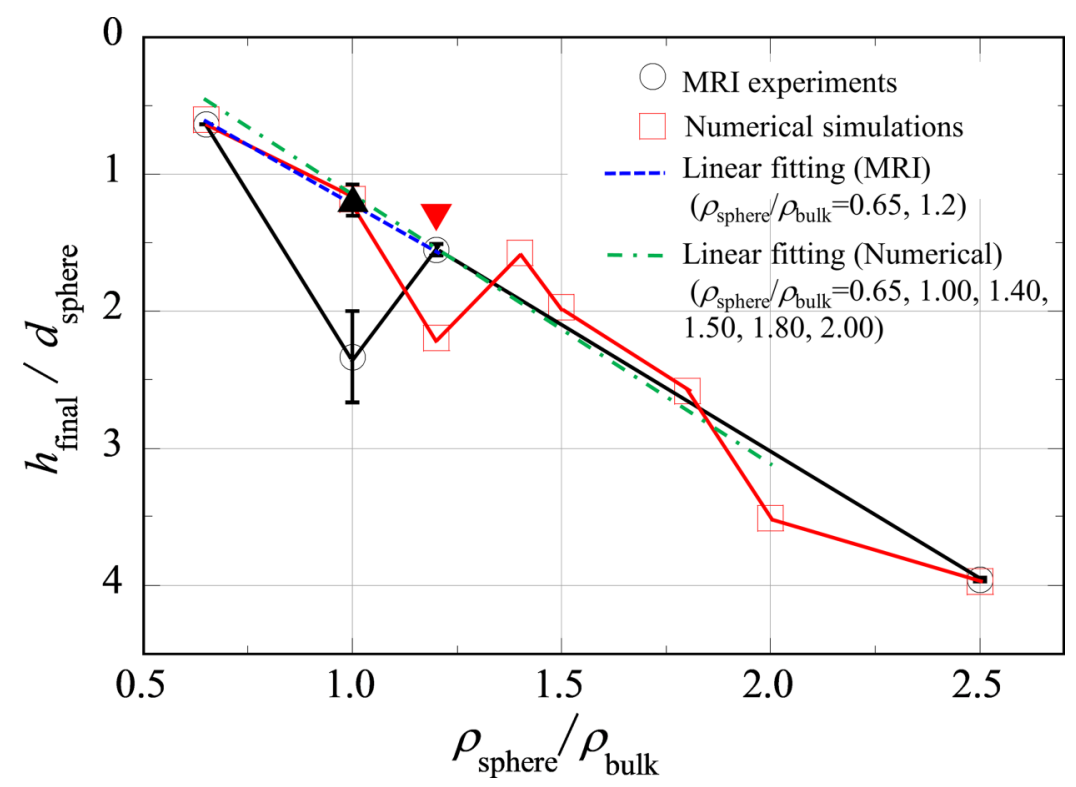

FIG. 9. Final sinking depths in MRI experiments and numerical simulations at $U_{0} / U_{\mathrm{mf}}=0.95$. The blue dashed and green dash-dotted lines show the linear least-squares fittings of the MRI results $\left(\rho_{\text {sphere }} / \rho_{\text {bulk }}=0.65\right.$ and 1.20) and numerical results $\left(\rho_{\text {sphere }} / \rho_{\text {bulk }}=0.65,1.00,1.40,1.50,1.80,2.00\right)$, excluding the anomalous results, respectively. The triangle and inverted triangle show the sinking depths reached at the first stop (step II) in the MRI experiment $(t=0.28 \mathrm{~s})$ and numerical simulation $(t=0.29 \mathrm{~s})$ that will appear in Fig. 14, respectively.

distribution around a sphere, are the dominating forces (besides gravity) in the floating regime $[47,48]$. The gas pressure gradient experienced by the sphere is slightly attenuated when bubbles go past the sides of the sphere; repeated bubble detachments from the sphere surface lead to an

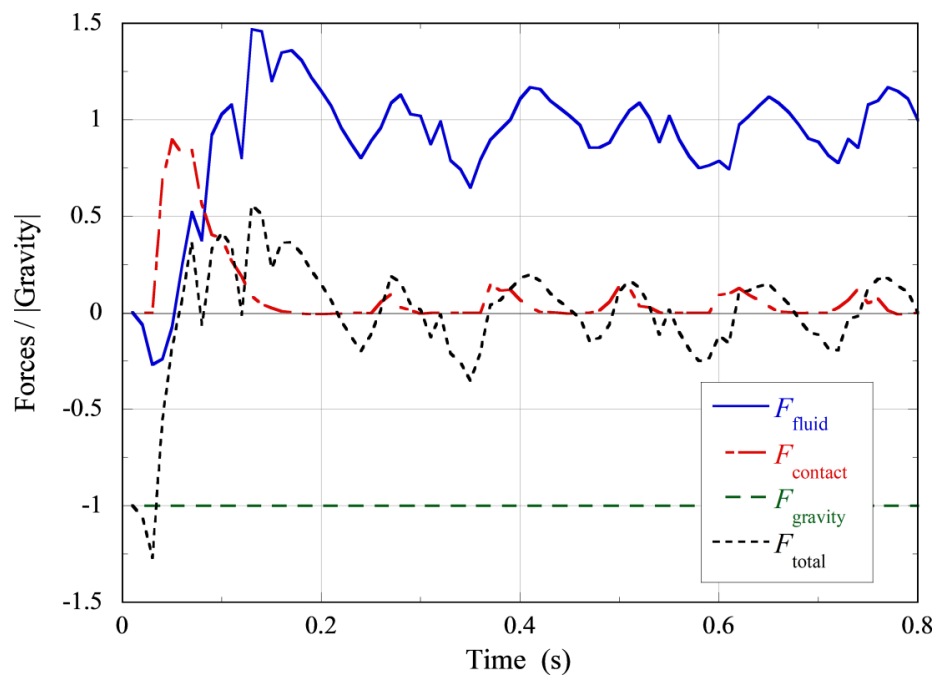

FIG. 10. Vertical forces acting on a floating sphere $\left(\rho_{\text {sphere }} / \rho_{\text {bulk }}=0.65, U_{0} / U_{\mathrm{mf}}=0.95\right)$. A positive force acts vertically upward. 


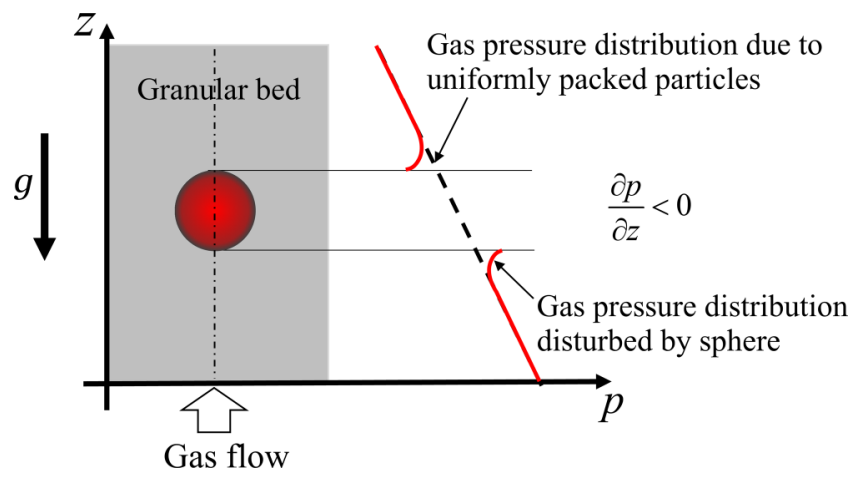

FIG. 11. Schematic of the gas pressure drop in a uniformly packed granular bed with a forced gas flow. A sphere submerged in such a particle bed experiences a vertical, upward-directed fluid force due to the negative pressure gradient in the bed. The black dashed line shows the gas pressure distribution in an undisturbed bed. The red solid line shows a gas pressure distribution disturbed by the presence of a submerged sphere.

oscillatory cycle of a small drop in fluid force, subsequent sphere sinking, and "reacquisition" of fluid force with sinking depth, followed by rerising to the surface and a new bubble detachment event. As a result of these subprocesses, the spheres keep floating near the bed surface on average. Related discussions on the reduction of fluid force due to bubble detachments will be developed in Sec. III B 3. In the fully fluidized regime $\left(U_{0}>U_{\mathrm{mf}}\right)$, a submerging of intruding objects due to the accumulation of splashed particles on its top has been reported [49]. In contrast, Fig. 10 shows that the net contact force is either acting vertically upward or close to zero, but not pointing vertically downward.

\section{Rapid sinking of heavy spheres $\left(\rho_{\text {sphere }} / \rho_{\text {bulk }}>1\right)$}

Figure 12 plots the vertical forces acting on a sphere with $\rho_{\text {sphere }} / \rho_{\text {bulk }}=1.80$ and the sinking velocity of the sphere as a function of time. The heavy sphere sinks immediately after having been released and stops in the middle of the bed [Fig. 4(b)]. As in the floating case, the contact forces

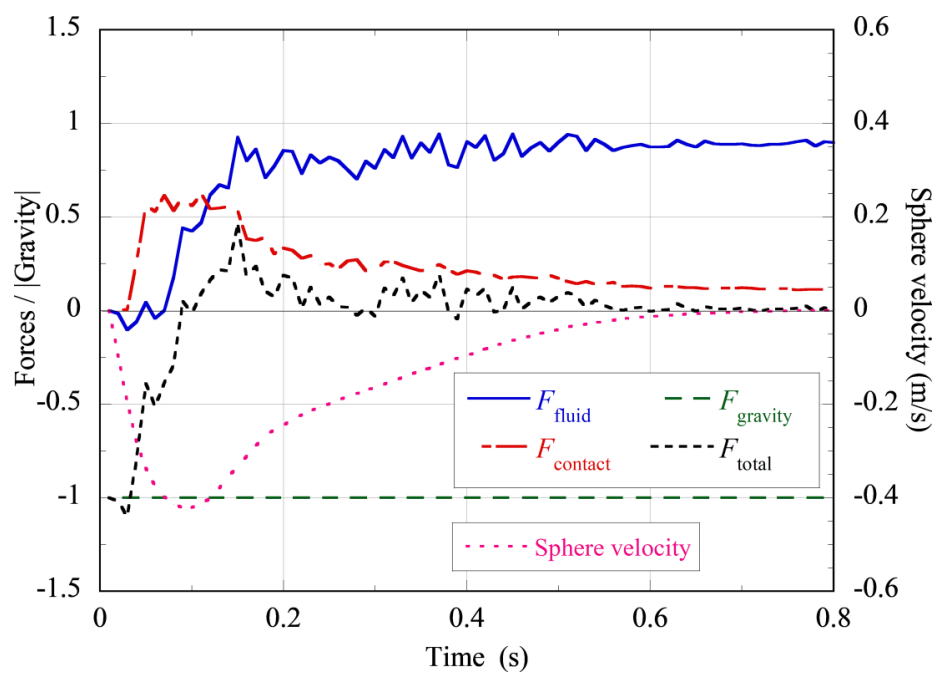

FIG. 12. Vertical forces and velocity of a rapidly sinking sphere $\left(\rho_{\text {sphere }} / \rho_{\text {bulk }}=1.80, U_{0} / U_{\mathrm{mf}}=0.95\right)$. 

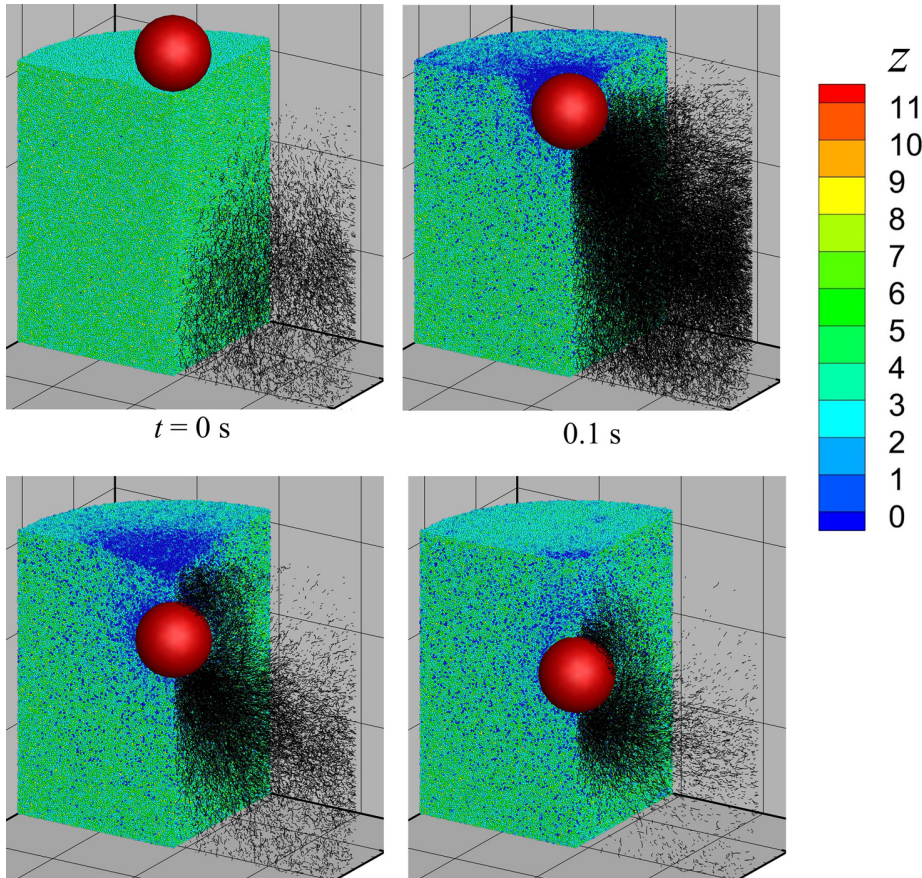

$0.2 \mathrm{~s}$

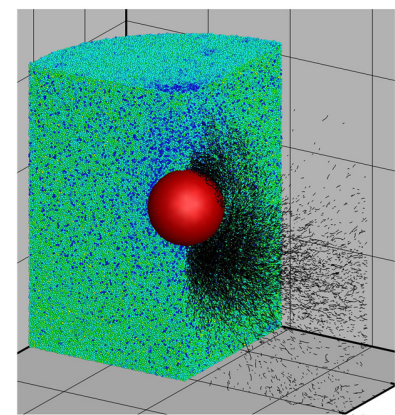

$0.3 \mathrm{~s}$

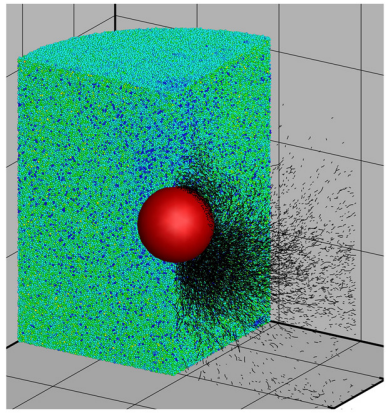

$0.4 \mathrm{~s}$

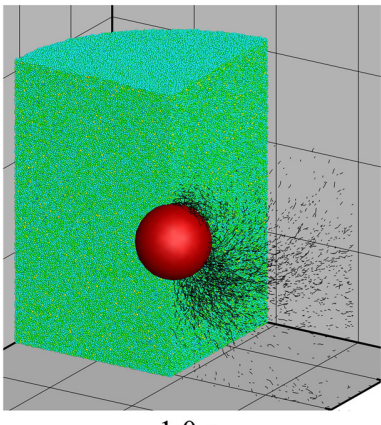

$1.0 \mathrm{~s}$

FIG. 13. Coordination number $z$ and force chains of normal contact forces $f_{\mathrm{C}, \mathrm{n}}$ scaled by the gravitational force working on a particle. The coordination number shows the number of particles and spheres in contact. Only force chains with $\left|\boldsymbol{f}_{\mathrm{C}, \mathrm{n}}\right| /\left|m_{\mathrm{p}} \boldsymbol{g}\right|>19.92$ are depicted $\left(\rho_{\text {sphere }} / \rho_{\text {bulk }}=1.80, U_{0} / U_{\mathrm{mf}}=0.95\right)$.

show a rapid increase after the sphere's impact with the bed surface. The sphere experiences an acceleration in the downward direction until $t \approx 0.095 \mathrm{~s}$. With increasing sinking depth of the sphere in the bed, the fluid force due to the gas pressure gradient increases and becomes approximately constant after $t \approx 0.6 \mathrm{~s}$.

In Fig. 13, coordination number and force chain are depicted. Corresponding to the rapid increase of net contact forces in the initial time of the sphere penetration $(t<0.1 \mathrm{~s}$ in Fig. 12), force chains are developed from the sphere bottom to a wide area of the bed. Energy dissipation due to the contacts as well as the development of fluid force attenuate the force chains. Once the sphere has stopped moving, the force chains have reduced further but still remain. Continuous contact between the bed particles and the sinking spheres at the bottom of the sphere prevents the formation and detachment of bubbles at the perimeter of heavy spheres. 


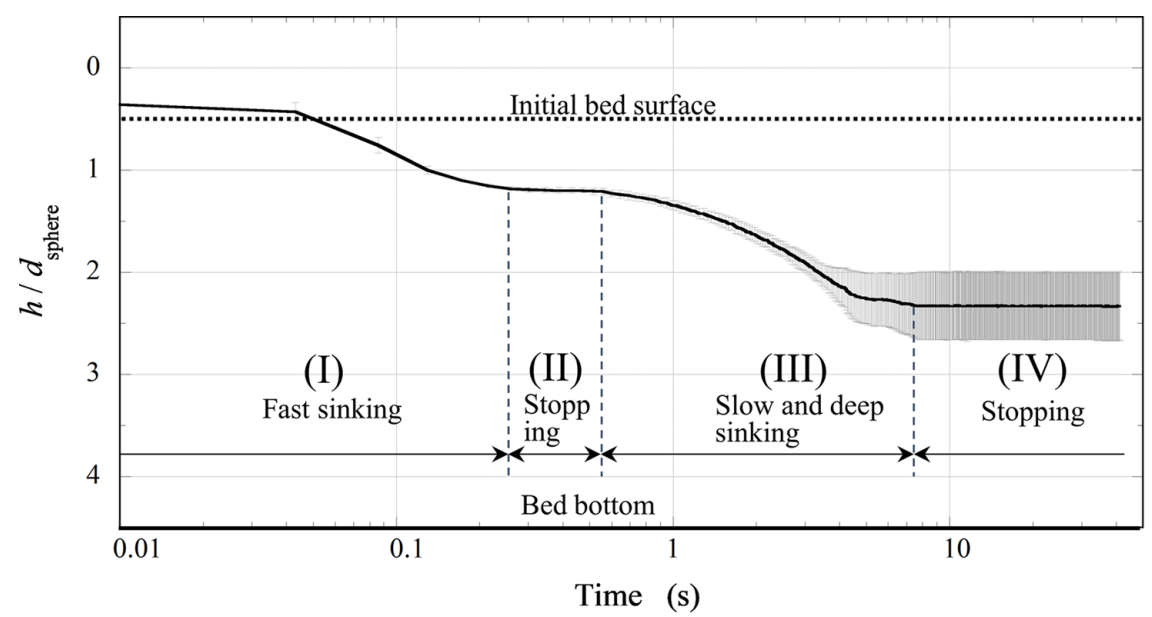

(a) MRI experiment $\left(\rho_{\text {sphere }} / \rho_{\text {bulk }}=1.00\right)$

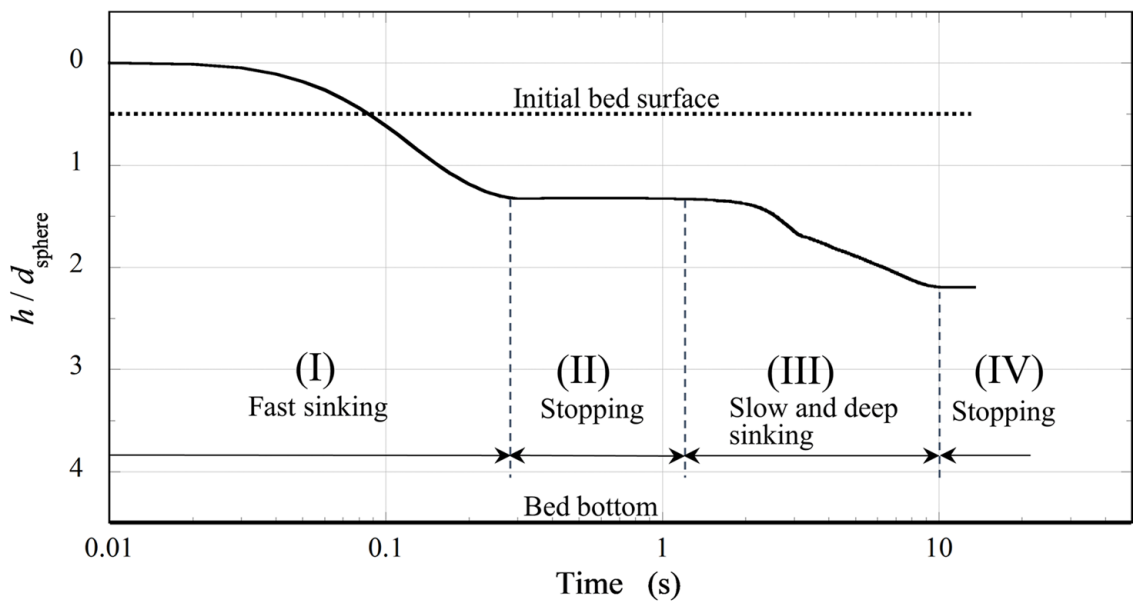

(b) Numerical simulation $\left(\rho_{\text {sphere }} / \rho_{\text {bulk }}=1.20\right)$

FIG. 14. Semilog plot of the trajectories of slow and deep sinking spheres $\left(U_{0} / U_{\mathrm{mf}}=0.95\right)$. The data are the same as shown in Fig. 4, but plotting on the spheres with $\rho_{\text {sphere }} / \rho_{\text {bulk }}=1.00$ for the MRI experiment and $\rho_{\text {sphere }} / \rho_{\text {bulk }}=1.20$ for the numerical simulation. The slow and deep sinking occurs in step III after it stopped once at step II.

\section{Anomalous slow and deep sinking of near to neutral buoyant spheres $\left(\rho_{\text {sphere }} / \rho_{\text {bulk }} \approx 1\right)$}

Figure 14 shows a semilog plot of sinking trajectories of slow and deep sinking spheres (Fig. 4, $\rho_{\text {sphere }} / \rho_{\text {bulk }}=1.00$ in the MRI experiment and 1.20 in the numerical simulation). From the MRI results [Fig. 14(a)], the sinking progress can be divided into four steps. In step I, after its release the sphere sinks quickly into the bed $(t<0.28 \mathrm{~s})$. In step II, the sphere is at rest $(0.28 \leqslant t<0.57$ $\mathrm{s})$, and, in step III, the sphere sinks further slowly $(0.57 \leqslant t<7.8 \mathrm{~s})$. In step IV, the sphere stops again $(t \geqslant 7.8 \mathrm{~s})$. Although we continued the observations until $t=40 \mathrm{~s}$, no further sinking was observed after it reached step IV. The fluidization-sensitive MRI image shown in Fig. 6(b) provides a snapshot of the sphere in step (I), while the MRI images in Fig. 6(c) are taken during step III. The two-step sinking process including a brief stop during step II is unique to the anomalous case 
( $\rho_{\text {sphere }} / \rho_{\text {bulk }} \approx 1.00$ ). The numerically determined sinking behavior [Fig. 14(b)] shows a great similarity to the experiment [Fig. 14(a)]: (I) fast sinking $(t<0.29 \mathrm{~s})$; (II) brief stopping $(0.29 \leqslant$ $t<1.20 \mathrm{~s}$ ); (III) slow and deep sinking $(1.20 \leqslant t<11.20 \mathrm{~s})$; (IV) final stopping $(t \geqslant 11.20 \mathrm{~s})$.

Figure 15 (a) plots the vertical forces acting on a sphere with $\rho_{\text {sphere }} / \rho_{\text {bulk }}=1.20$ obtained numerically; the inset magnifies the data during the initial stage of the sinking process $(t<0.5 \mathrm{~s})$. Up to the end of step I $(t<0.29 \mathrm{~s})$, the contribution of the forces acting on a close to neutrally buoyant sphere is similar to light (Fig. 10) and heavy spheres (Fig. 12): The force due to particle contacts appears just after the impact of the intruder into the bed; the fluid force increased with a slight delay compared to the contact force. In Fig. 9, the sphere sinking depths reached at the first stop in step II are also plotted. In particular, the MRI result collapsed well with the linear approximation, suggesting the reachable depths due to the inertia of spheres are linearly dependent on the sphere density even in the case of near to neutrally buoyant spheres. After $t=0.2 \mathrm{~s}$, the net contact force becomes small compared to the other forces and indeed becomes even negative [Fig. 15(a)]; a clear difference exists comparing to the floating and the rapid sinking cases discussed previously. Figure 16 shows the coordination number and force chains. In contrast to the heavy case (Fig. 13), the force chains are not concentrated on the bottom, but on the top part of the sphere. We also recognize from Fig. 15(a) that the magnitude of the upward-directed fluid force slightly exceeds gravity. The total force acting on the intruding sphere is enlarged in Fig. 15(b), showing that the forces acting on the sphere are with fluctuations ( $\leqslant \pm 9 \%$ of gravity) but close to being fully balanced $\left(F_{\text {total }} \approx 0\right)$ during the slow and deep sinking in step III. These results allow us to propose that in step III the sphere is pushed up by the fluid force as its magnitude is slightly larger than that of gravity, but, at the same time, it is pushed back by the contacts in its cap region. Figure 15 (c) plots the velocity of the sphere in the vertical direction. Following a rapid downward motion in the initial stage $(t<0.3 \mathrm{~s}$; see the inset), the velocity rapidly decreases with quasiperiodic fluctuations until the sphere stops $(t=11.20 \mathrm{~s})$. Magnified views of the velocity and forces acting on the sphere during the time range $t=2.6$ to $3.0 \mathrm{~s}$ (typical period in step III and highlighted in Fig. 15) are plotted in Fig. 17. To "promote" the sinking motion, the force equilibrium has to be "broken" by either a reduction of the upward-directed fluid force or an increase of the downward-directed net contact force or both effects together. As highlighted in Fig. 17, the occurrence of negative total forces (resulting in a sphere sinking) is synchronized with a decrease of fluid forces and not an increase in the net contact forces. It is also noted that, even when the fluid forces are decreased, their magnitude is still slightly larger than gravity. Hence, a downward motion of the sphere is ultimately induced by the net contact forces, which are always acting downward during the slow and deep sinking period (step III).

Snapshots of the local void fraction and gas pressure distribution in a vertical center plane for a time period when the total force acting on the sphere becomes negative $(t=2.91-2.96 \mathrm{~s}$, surrounded by dashed lines in Fig. 17) are given in Fig. 18(a). As previously discussed (Fig. 11), because the sphere disturbs the gas flow field, the pressure isolines are not always perpendicular to the vertical direction, but are bent near the surface of the sphere. As a result, a form (pressure) drag force is acting on the sphere in addition to the buoyant force that results from the background pressure gradient due to the particle bed. The form drag could explain why the magnitude of the fluid force becomes slightly larger than the gravitational force, even for a near to neutrally buoyant sphere $\left(\rho_{\text {sphere }} \approx \rho_{\text {bulk }}\right)$. In Fig. 18(a), we can observe the growth and detachment of a bubble at the bottom hemisphere of the sphere. Because the gas pressure inside a bubble is almost uniform [15], a slight attenuation of the gas pressure gradient will be experienced by the sphere when a detached bubble passes next to the sphere. Figure 18(b) shows the corresponding particle velocities in the vertical direction, downward motion of particles are clearly observable at the cap region and also at the bottom region of the sphere to a small extent. Only typical results were shown in Figs. 17 and 18; the bubble formation and detachment are repeated quasiperiodically during the slow and deep sinking in step III (Fig. 8(c) and Supplemental Video 5 [44]). Due to the small deviations from the force equilibrium, the sphere sink proceeded very slowly.

Our experiments and simulations, however, do not explain why the sphere comes to a complete standstill in step II prior to the onset of the slow and deep sinking. A possibility is that local 


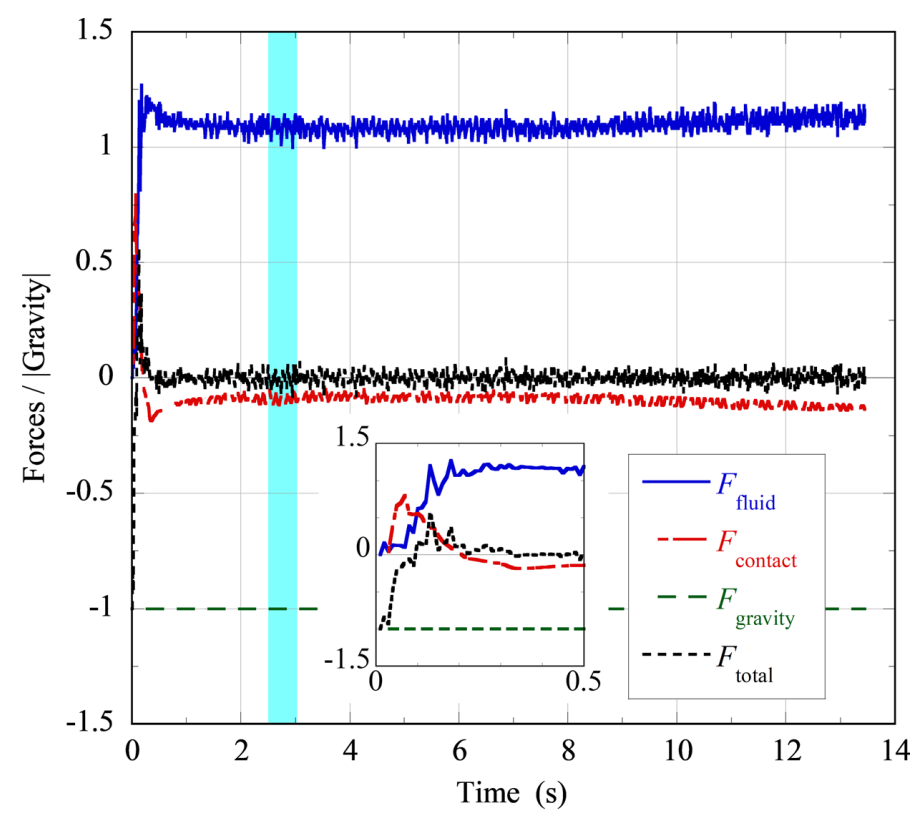

(a) Vertical forces

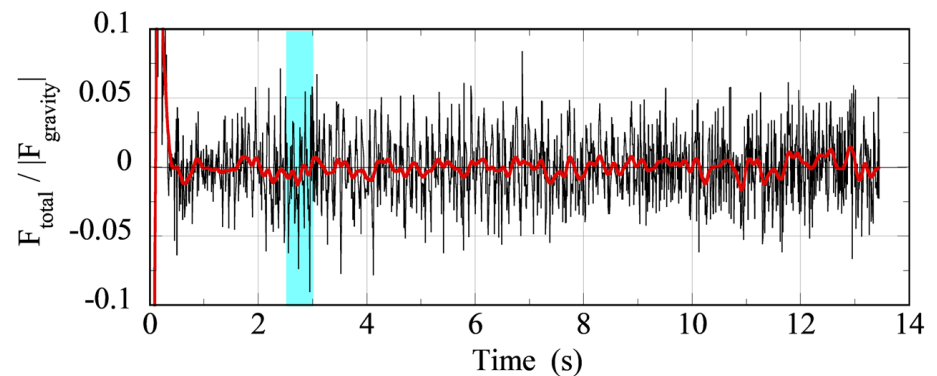

(b) Total force

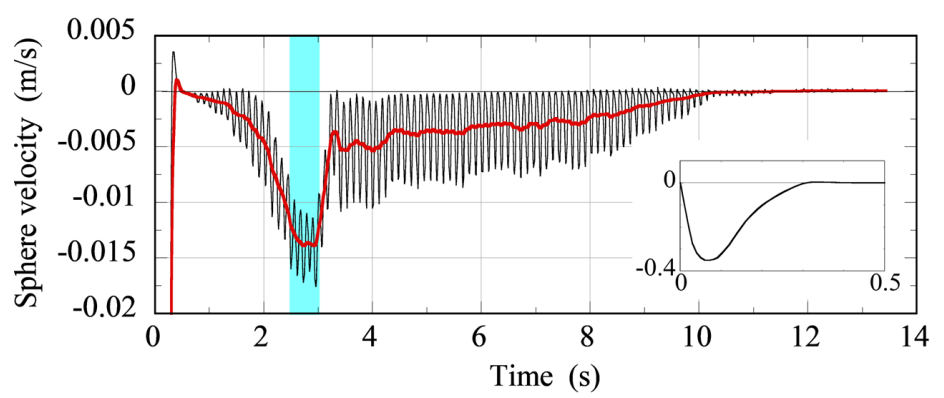

(c) Sphere velocity

FIG. 15. (a) Vertical forces acting on a slow and deep sinking sphere $\left(\rho_{\text {sphere }} / \rho_{\text {bulk }}=1.20, U_{0} / U_{\text {mf }}=0.95\right)$. (b) Enlarged view of the total force. (c) Sphere sinking velocity as a function of time. In (b), (c), the black lines plot the simulation results and the red lines plot the results smoothed by a Gaussian filter. Note that the velocity range in (c) is very different from that in Fig. 12. The insets in (a), (c) show a magnification of the data in the initial stage of the sinking process $(t \leqslant 0.50 \mathrm{~s})$. For the highlighted section $(t=2.6-3.0 \mathrm{~s})$, further detailed discussions will be developed later. 


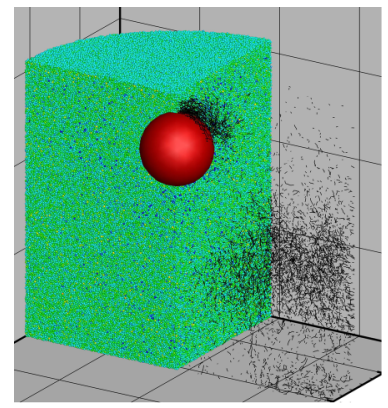

$t=1.0 \mathrm{~s}$

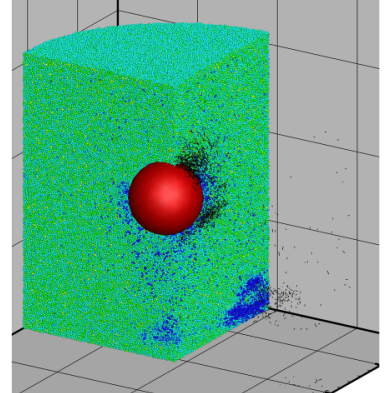

$9.0 \mathrm{~s}$

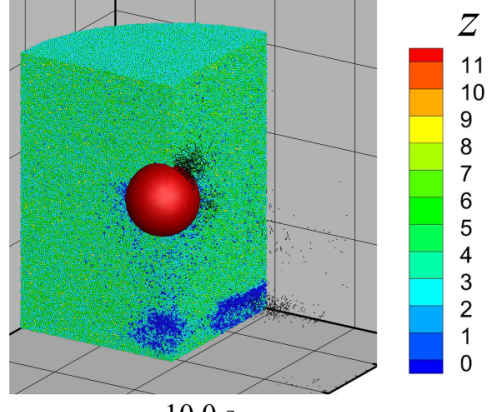

$10.0 \mathrm{~s}$

FIG. 16. Coordination number $z$ and force chains of normal contact forces $\left(f_{\mathrm{C}, \mathrm{n}}\right)$ scaled by the gravitational force working on a particle. The coordination number shows the number of particles and spheres in contact. Only force chains with $\left|\boldsymbol{f}_{\mathrm{C}, \mathrm{n}}\right| /\left|m_{\mathrm{p}} \boldsymbol{g}\right|>13.28$ are depicted $\left(\rho_{\text {sphere }} / \rho_{\text {bulk }}=1.20, U_{0} / U_{\mathrm{mf}}=0.95\right)$.
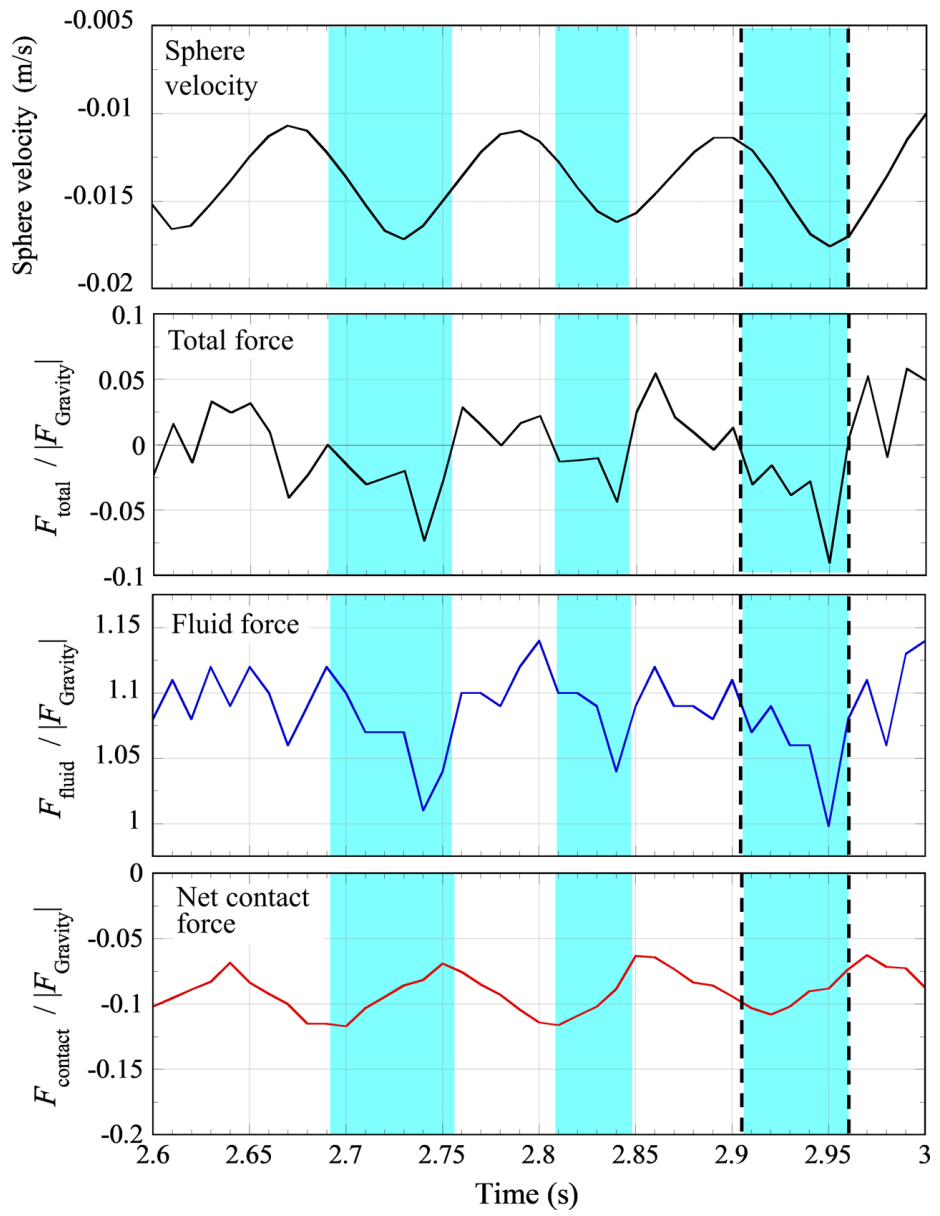

FIG. 17. Sphere velocity and forces acting on the sphere during $t=2.6-3.0 \mathrm{~s}\left(\rho_{\text {sphere }} / \rho_{\text {bulk }}=1.20\right.$, $\left.U_{0} / U_{\mathrm{mf}}=0.95\right)$. In the highlighted sections, the total force becomes negative and the sphere sinking velocity increases. 


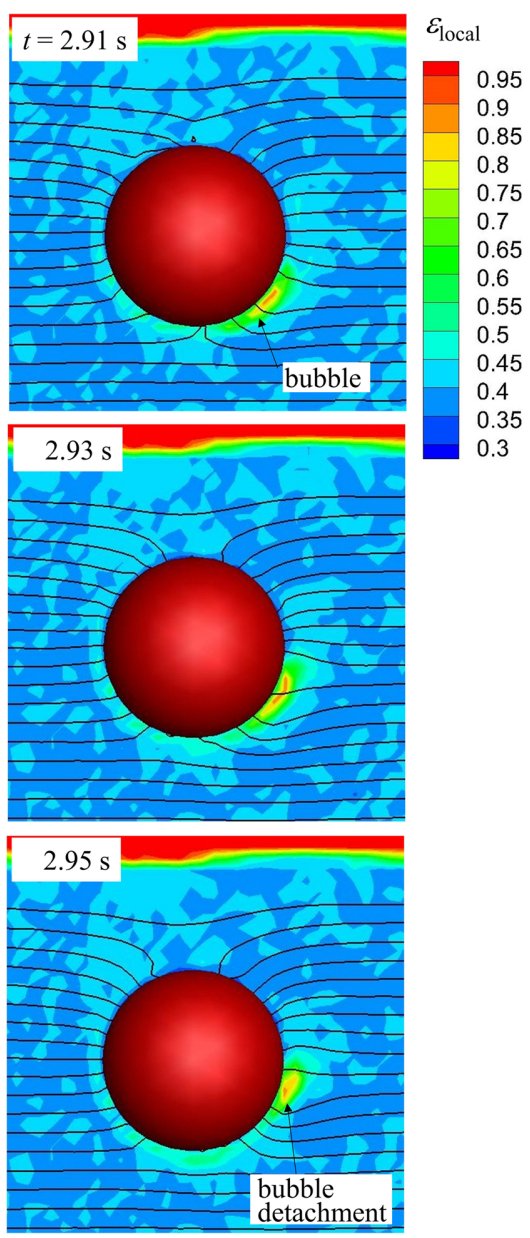

(a) Local void fraction and gas pressure
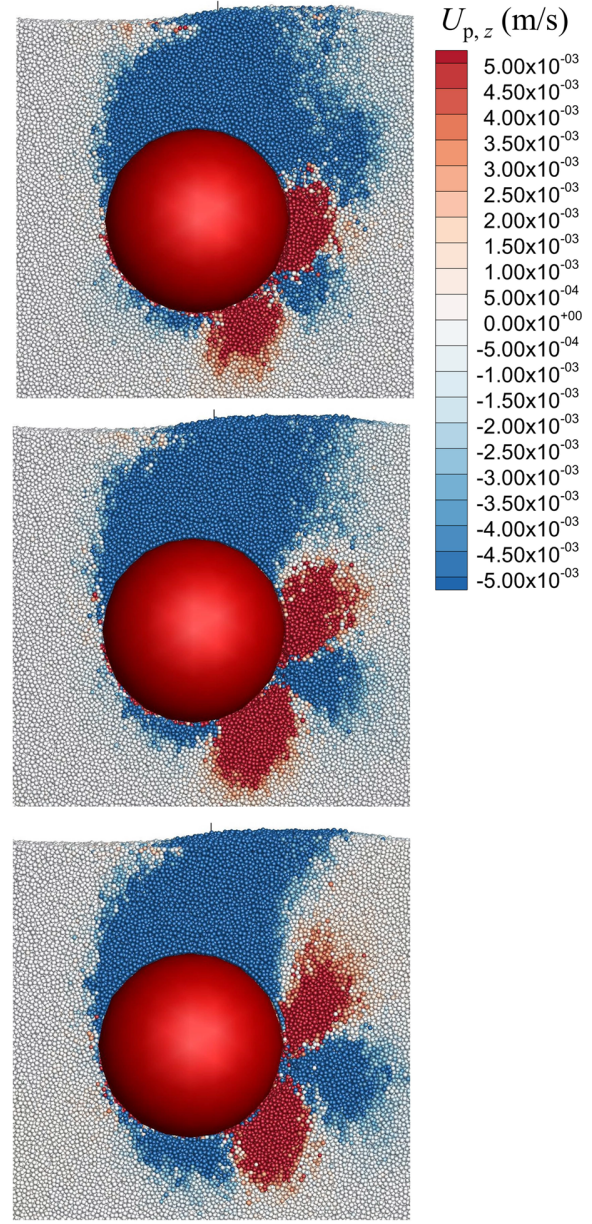

(b) Particle velocity in vertical direction

FIG. 18. (a) Distribution of the local void fraction and gas pressure in a vertical, central plane during $t=2.91-2.96 \mathrm{~s}\left(\rho_{\text {sphere }} / \rho_{\text {bulk }}=1.20, U_{0} / U_{\mathrm{mf}}=0.95\right)$. Gas pressure isolines are plotted with an interval $\Delta p=$ $25 \mathrm{~Pa}$. The detached bubble extends the distance between successive isolines near the sphere and induces a relaxation of the fluid force acting on the sphere. (b) Particle velocity in the vertical direction. Downward motion of particles occurs in the cap region at the top of the sphere. Upward particle motion occurs locally when a bubble detaches.

fluidization below and around the sphere does not form instantaneously but requires some time to form and to trigger the onset of the deep and slow sinking. This effect will be the topic of future research.

Next, we explored the question of what the mechanism is that leads to the ultimate stopping of a sphere, i.e., after the slow and deep sinking period. In Fig. 6(d), the fluidization-sensitive MRI measurement has shown a weakening of the bubbling activity and a shrinkage of the local fluidization region after the sphere has reached its final depth. In Fig. 15(c), the discrete particle simulation has shown a decrease of the fluctuations of the sinking velocity with the progress of sphere sinking. We propose that the stopping of the sphere sinking process is due to a weakening of the bubbling activity. There are two possible reasons for a reduced bubbling activity with increasing sinking depth. First, in the system we studied, the superficial velocity is slightly below the minimum 


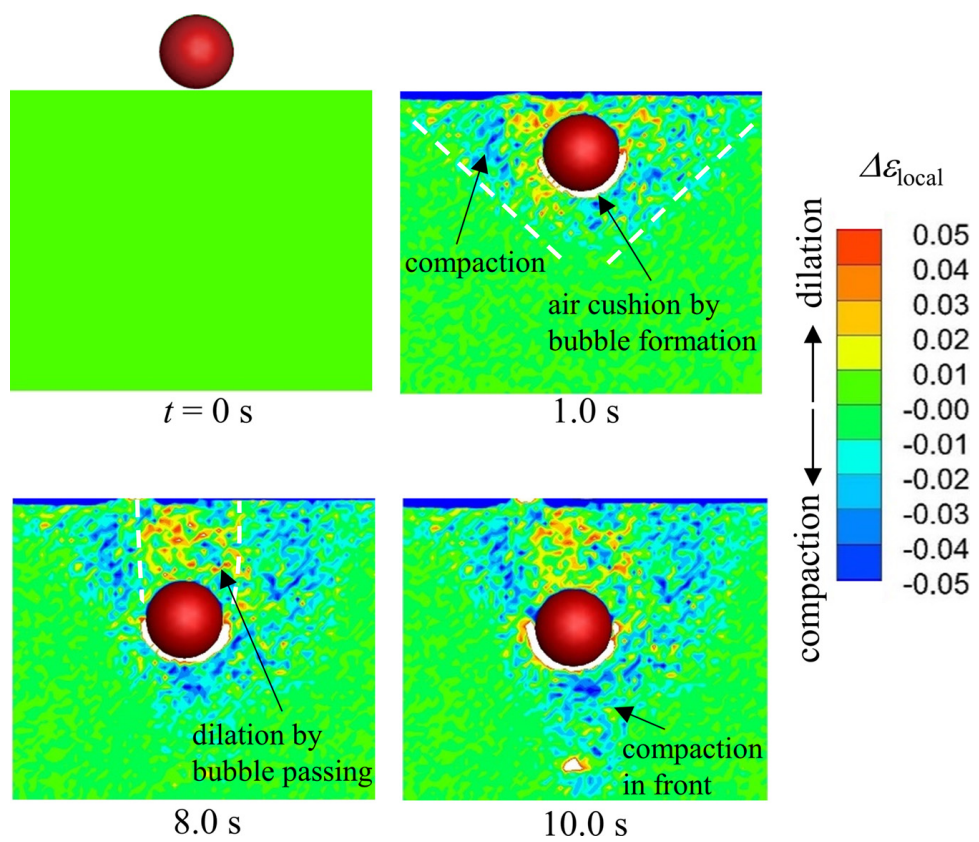

FIG. 19. Change in the local void fraction relative to the initial state $\Delta \varepsilon_{\text {local }}(t)=\varepsilon_{\text {local }}(t)-\varepsilon_{\text {local }}(t=0)$ ( $\left.\rho_{\text {sphere }} / \rho_{\text {bulk }}=1.20, U_{0} / U_{\mathrm{mf}}=0.95\right)$. The difference from the initial void fraction was calculated using the local void fraction in each CFD cell fixed in space. Only variations in the range of $\Delta \varepsilon_{\text {local }}= \pm 0.05$ are depicted. An "air cushion" exists always at the bottom of the sphere (see Supplemental Video 6 [44]).

fluidization velocity. Hence, the local particle pressure, which is similar to a hydrostatic pressure caused by the weight of the particles above a location and transmitted through contacts between particles, is small but still remains and may increase the bulk stiffness of the granular materials, which in turns weakens the extent of bubbling further down the bed. Second, the sinking of the sphere results in changes in the local packing arrangement. Royer et al. [14] reported the compaction of a granular packing at the front of an intruding sphere in a loose bed $\left(\varepsilon_{0}=0.49\right)$. Because our bed is close to the loose random packing limit of monosized particles, a similar compaction behavior is expected. Figure 19 depicts numerically obtained deviations of the local void fraction from the initial state $\left[\Delta \varepsilon_{\text {local }}(t)=\varepsilon_{\text {local }}(t)-\varepsilon_{\text {local }}(t=0)\right]$, where positive and negative values of $\Delta \varepsilon_{\text {local }}$ imply dilation and compaction, respectively. To obtain $\Delta \varepsilon_{\text {local }}(t)$, the local void fraction was determined by counting the volume of particles in each CFD cell fixed in space. For clarity, only the results in the range of $\Delta \varepsilon_{\text {local }}= \pm 0.05$ are shown in Fig. 19. After the impact of the sphere, a void fraction change occurred locally in an inverted cone-shaped region reaching from the front of the sphere to the bed surface $(t=1.0 \mathrm{~s})$. The shape of the region that is perturbed by the intruder sphere is very similar to the region in which local fluidization occurs during the initial impact [Fig. 6(b)]. Dilation occurred in a region around the sphere and compaction occurred in the remainder of the inverted cone-shaped region. The region at the bottom of the sphere, referred to as the "air cushion" here, exhibits higher void fractions $\left(\Delta \varepsilon_{\text {local }}>0.05\right)$ due to the formation of bubbles. During and after the local fluidization-induced, slow and deep sinking (e.g., $t=8.0 \mathrm{~s}$ and $10.0 \mathrm{~s}$ ), both the dilated and compacted regions expand with the increasing sinking depth of the sphere. Between the sphere and the bed surface, dilation is observed owing to the detachment and passage of bubbles. The shape of the dilated region is similar to that of the local fluidization region shown in Fig. 6(c). It is noted that the air cushion remains in front of the sphere, even after the sinking process has been stopped completely. The packing fraction is one of the main factors that influence the bulk stiffness of granular materials, in particular for slow granular flows. Gravish et al. 


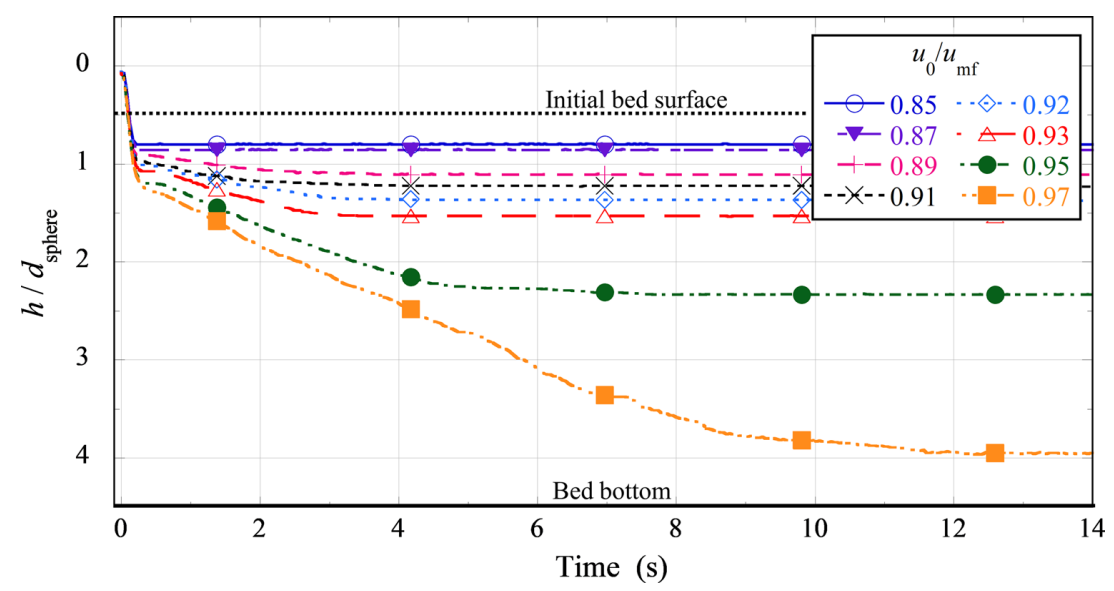

FIG. 20. MRI-determined sinking trajectories of $\rho_{\text {sphere }} / \rho_{\text {bulk }}=1.00$ spheres at different superficial velocities.

[50] demonstrated that a $6.9 \%$ increase in the initial packing fraction resulted in a $32 \%$ increase in the mean drag force acting on a vertical plate moving horizontally in a granular bed. Although their conditions are largely different from the system studied here, i.e., a forced gas flow in the present case, Fig. 19 reveals that local compaction values reach up to $\Delta \varepsilon=-0.05$ (i.e., an $8.4 \%$ increase from the initial packing fraction). These results indicate that the final stopping of the sphere in the center of the bed can be explained by a combination of a rising hydrostatic pressure with sinking depth and bed compaction in front of the sphere. These effects can increase the bulk stiffness of the packing, reduce in turn bubble formation and detachment, and hence stabilize the fluid force acting upward on the sphere.

\section{Dependence of sinking depth on superficial velocity}

Figures 20, 21, and 22 show the average sinking trajectories as determined by MRI for $\rho_{\text {sphere }} / \rho_{\text {bulk }}=1.00,1.20$, and 2.50 , respectively, with superficial velocities $U_{0} / U_{\text {mf }}$ varying between 0.85 and 0.97 . Figure 23 shows the final sinking depths depending on $U_{0} / U_{\mathrm{mf}}$. For all $\rho_{\text {sphere }} / \rho_{\text {bulk }}$ values, there is a monotonic increase in the final sink depth with increasing $U_{0} / U_{\mathrm{mf}}$. A

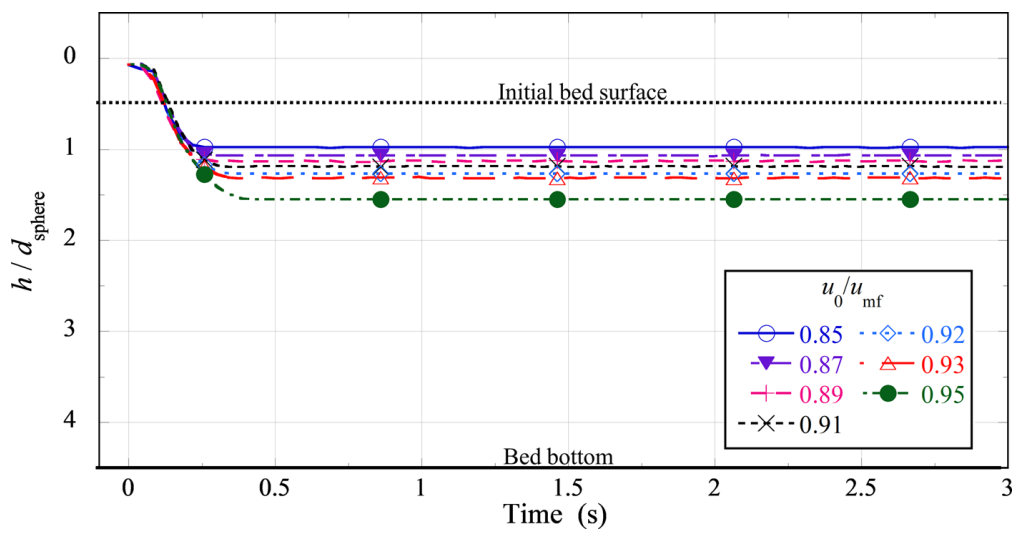

FIG. 21. MRI-determined sinking trajectories of spheres with $\rho_{\text {sphere }} / \rho_{\text {bulk }}=1.20$ for different superficial velocities. 


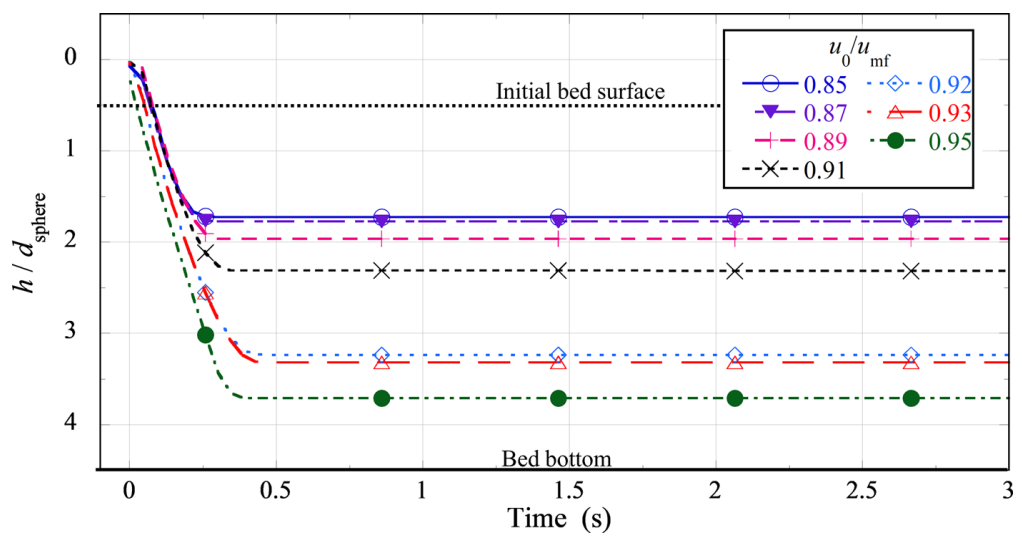

FIG. 22. MRI-determined sinking trajectories of spheres with $\rho_{\text {sphere }} / \rho_{\text {bulk }}=2.50$ for different superficial velocities.

comparison between $\rho_{\text {sphere }} / \rho_{\text {bulk }}=1.00$ and 1.20 shows that the anomalous slow and deep sinking occurs when $U_{0} / U_{\mathrm{mf}}>0.89$. In Fig. 23, sinking depths reached at the first stop are also plotted for $\rho_{\text {sphere }} / \rho_{\text {bulk }}=1.00$. The two-step sinking, as described in Fig. 14, can be observed in all of the cases with $U_{0} / U_{\mathrm{mf}} \geqslant 0.89$. The duration and sinking depth of the local fluidization-induced, slow and deep sinking are very sensitive to $U_{0} / U_{\mathrm{mf}}$. When $U_{0} / U_{\mathrm{mf}}$ increases, the gas pressure drop across the bed also increases, and the stiffness of the granular materials is reduced. This, in turn, facilitates

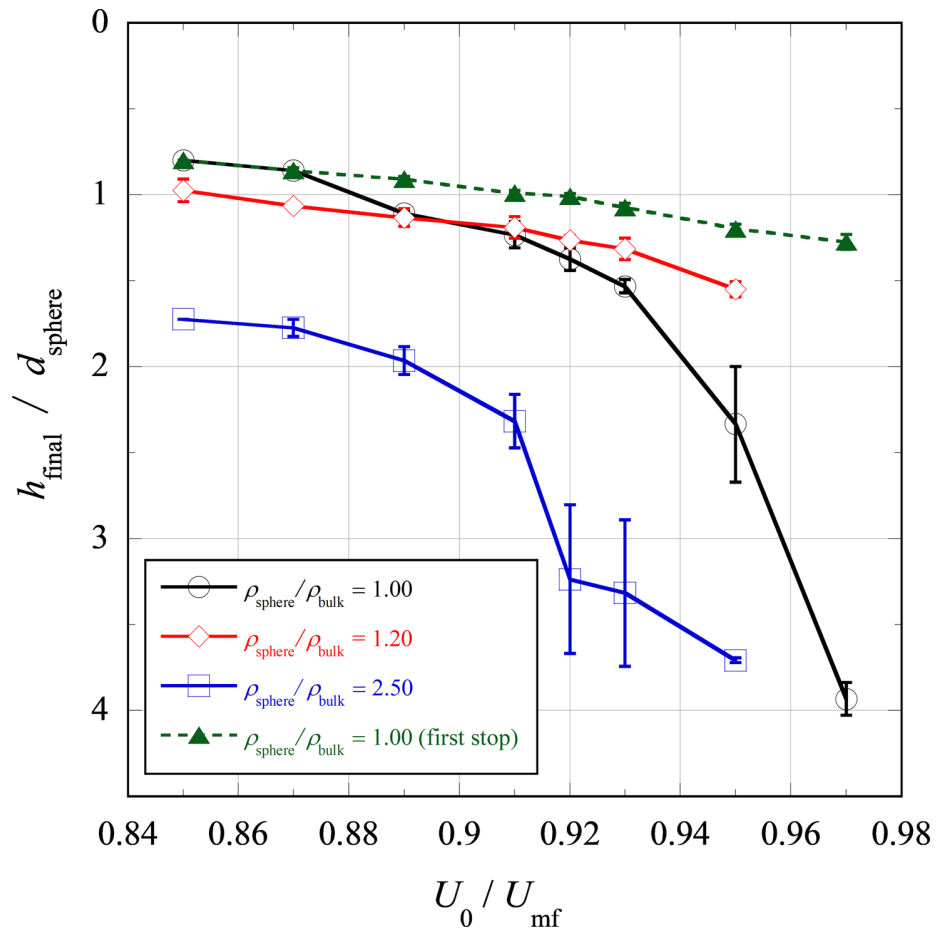

FIG. 23. MRI-determined final sinking depths depending on superficial velocity. The triangle shows the sinking depths reached at the first stop $\left(\rho_{\text {sphere }} / \rho_{\text {bulk }}=1.00\right)$. 
the formation and detachment of local bubbles at deeper positions, and the sinking proceeds further as $U_{0} / U_{\mathrm{mf}}$ increases.

\section{CONCLUSIONS}

Anomalous sinking of an intruding sphere in a granular packing aerated slightly below the minimum fluidization velocity was investigated by real-time MRI experiments and discrete particle simulations. Despite differences in the bed particles, major findings obtained in a previous study [20] were reproduced experimentally and numerically, and the mechanisms of the anomalous sinking were elucidated. We found the following:

(1) Inertial and local fluidization-induced slow and deep sinking exist. Regardless of the sphere density, the sinking of the sphere due to the inertia of the sphere proceeded quickly after its release. The sinking depth obtained in this step increases monotonically with increasing sphere density. After a brief interruption, the local fluidization-induced, slow and deep sinking occurred, but only when the sphere density was similar to the bulk density of the bed $\left(\rho_{\text {sphere }} / \rho_{\text {bulk }} \approx 1\right)$.

(2) Attenuation of the gas pressure gradient induces the slow and deep sinking. During the local fluidization-induced, slow and deep sinking, both a local fluidization and the formation and detachment of bubbles from the sphere surface were observed. Contrary to our expectations, the net contact force acting on the sphere is directed downward during sinking. A force balance analysis revealed that the upward-directed fluid force due to the gas pressure gradient is almost fully counterbalanced by the sum of the net contact force and the gravitational force. When a bubble is detached from the sphere's surface, the gas pressure gradient experienced by the sphere is slightly attenuated and results in a reduction of the upward-directed fluid force. As a result, the sphere is pushed downward by the particle cap that is formed on top of the sphere and sphere sinking proceeds. Bubble formation and detachment are quasiperiodic processes during this sinking motion. The sinking velocity is very slow because the deviations from the force equilibrium are small. No bubble formation was observed for heavier spheres $\left(\rho_{\text {sphere }} / \rho_{\text {bulk }}>1.0\right)$ during sinking; hence, local bubbling plays a critical role in driving the sinking anomaly.

(3) Sphere stopping is caused by a weakening of local bubbling. Stopping of the sphere after the local fluidization-induced, slow and deep sinking is attributed to the weakening of the local bubbling due to two possible factors: (i) remaining hydrostatic particle pressure in the bed, and (ii) local compaction caused by the sphere sinking. Both of these can lead to an increased bulk stiffness of the packing and reduce in turn the tendency for bubble formation and detachment. This results in a stabilization of the upward-directed fluid force at deeper sphere positions. It is also worth noting that a region of local fluidization with some bubble formations remained even after the sphere had reached its final depth.

(4) Real-time MRI can probe the dynamic response of a granular packing. The sinking dynamics of intruder objects in a granular packing aerated slightly below the minimum fluidization velocity is very complex and it is hardly explainable from macroscopic considerations. MRI is a powerful tool to understand better the dynamics of sphere sinking, local bubbling, and fluidization in visually opaque granular systems. In particular, fluidization-sensitive MRI enables us to characterize the spatiotemporal response of a partially fluidized system.

\section{ACKNOWLEDGMENTS}

We would like to thank Murino Kobayakawa for stimulating discussions. T.T. and T.T. acknowledge partial funding by the Japan Society for the Promotion of Science KAKENHI under Grant No. JP16K06081; A.P., C.R.M., and K.P.P. acknowledge partial funding by the Swiss National Science Foundation under Grant No. 200020_182692. All computations were performed using the Cray XC-40 supercomputer at the Academic Center for Computing and Media Studies, Kyoto University. 


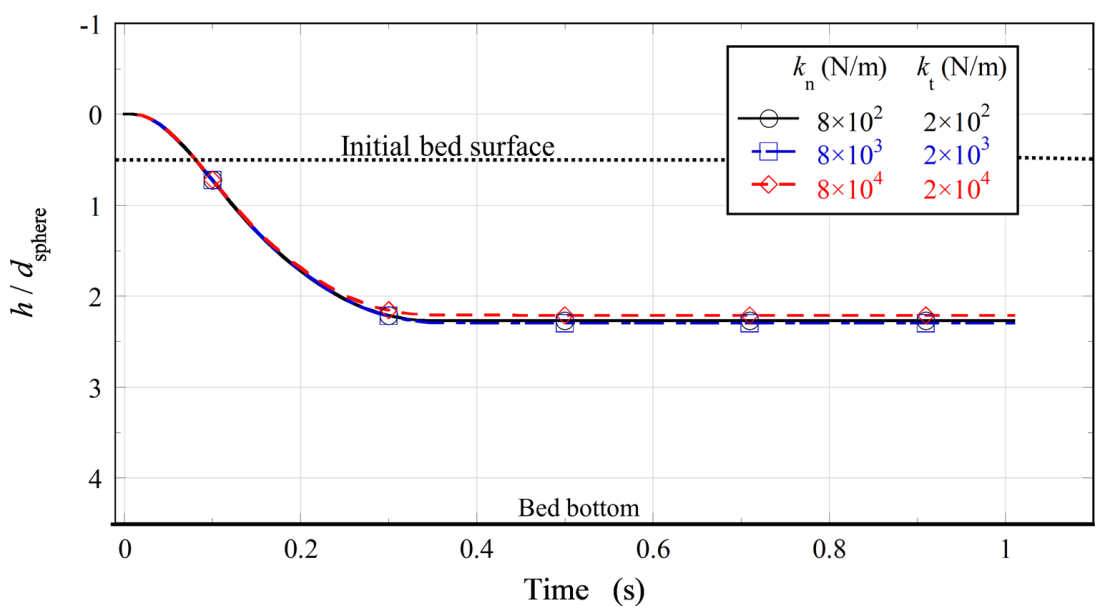

FIG. 24. Numerical results of the sinking depths of a sphere as a function of the spring constants $\left(U_{0} / U_{\mathrm{mf}}=\right.$ $\left.0.95, \rho_{\text {sphere }} / \rho_{\text {bulk }}=2.50, \varepsilon_{0}=0.398\right)$.

\section{APPENDIX A: DEPENDENCE OF THE SINKING DEPTH ON THE SPRING CONSTANT}

Figure 24 shows numerical results of the sinking depth of a sphere as a function of the magnitude of the spring constant. Both the normal, $k_{\mathrm{n}}$, and tangential, $k_{\mathrm{t}}$, spring constants were varied over three orders of magnitude. We have set $U_{0} / U_{\mathrm{mf}}=0.95$ and $\rho_{\text {sphere }} / \rho_{\text {bulk }}=2.5$. The initial void fraction of the packings was $\varepsilon_{0}=0.398$ regardless of the spring constant. No dependence of the sinking depth on the spring constant is observed in Fig. 24.

\section{APPENDIX B: DEPENDENCE OF THE SINKING DEPTH ON THE COEFFICIENT OF FRICTION}

Figure 25 plots the numerical results of the sinking depth of a sphere sinking as a function of the coefficient of friction, varying $\mu_{\mathrm{p}}$ between 0.30 and 0.70 at $U_{0} / U_{\mathrm{mf}}=0.94$ and $\rho_{\text {sphere }} / \rho_{\text {bulk }}=1.20$. The other conditions, including the initial void fraction, were the same as those reported in Sec. II C. Except at $\mu_{\mathrm{p}}=0.30$ and 0.31 , the calculations were terminated at $t=4.5 \mathrm{~s}$ because no further sinking was observed.

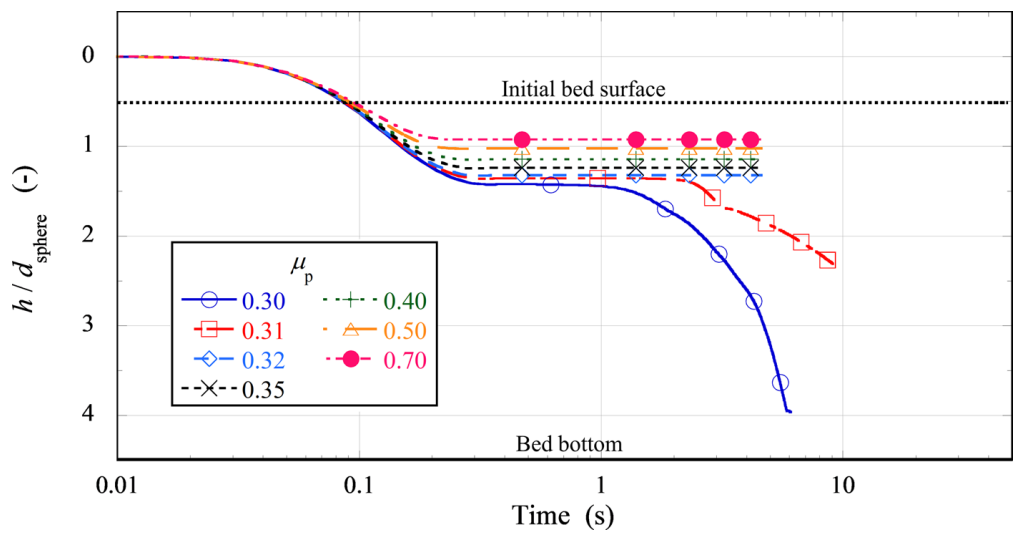

FIG. 25. Numerical results of the sinking depths of a sphere as a function of the coefficient of friction $\mu_{\mathrm{p}}$ $\left(U_{0} / U_{\mathrm{mf}}=0.94, \rho_{\text {sphere }} / \rho_{\text {bulk }}=1.20, \varepsilon_{0}=0.403\right)$. 
[1] H. M. Jaeger, S. R. Nagel, and R. P. Behringer, Granular solids, liquids, and gases, Rev. Mod. Phys. 68, 1259 (1996).

[2] A. J. Liu and S. R. Nagel, Jamming is not just cool any more, Nature 396, 21 (1998).

[3] R. M. Nedderman, Statics and Kinematics of Granular Materials (Cambridge University Press, Cambridge, 1992).

[4] K. K. Rao and P. R. Nott, An Introduction to Granular Flow (Cambridge University Press, Cambridge, 2008).

[5] B. Andreotti, Y. Forterre, and O. Pouliquen, Granular Media between Fluid and Solid (Cambridge University Press, Cambridge, 2013).

[6] H. Katsuragi, Physics of Soft Impact and Cratering (Springer Japan, Tokyo, 2016).

[7] H. K. Pak and P. R. Behringer, Bubbling in vertically vibrated granular-materials, Nature 371, 231 (1994).

[8] S. Matsusaka, M. Kobayakawa, M. Mizutani, M. Imran, and M. Yasuda, Bubbling behavior of a fluidized bed of fine particles caused by vibration-induced air inflow, Sci. Rep. 3, 1190 (2013).

[9] N. Burtally, P. J. King, and M. R. Swift, Spontaneous air-driven separation in vertically vibrated fine granular mixtures, Science 295, 1877 (2002).

[10] C. Zeilstra, M. A. Van Der Hoef, and J. A. M. Kuipers, Simulation study of air-induced segregation of equal-sized bronze and glass particles, Phys. Rev. E 74, 010302(R) (2006).

[11] P. Biswas, P. Sánchez, M. R. Swift, and P. J. King, Numerical simulations of air-driven granular separation, Phys. Rev. E 68, 050301(R) (2003).

[12] M. Klein, L. L. Tsai, M. S. Rosen, T. Pavlin, D. Candela, and R. L. Walsworth, Interstitial gas and density segregation of vertically vibrated granular media, Phys. Rev. E 74, 010301(R) (2006).

[13] J. R. Royer, E. I. Corwin, P. J. Eng, and H. M. Jaeger, Gas-Mediated Impact Dynamics in Fine-Grained Granular Materials, Phys. Rev. Lett. 99, 038003 (2007).

[14] J. R. Royer, B. Conyers, E. I. Corwin, P. J. Eng, and H. M. Jaeger, The role of interstitial gas in determining the impact response of granular beds, Europhys. Lett. 93, 28008 (2011).

[15] R. Jackson, The Dynamics of Fluidized Particles (Cambridge University Press, Cambridge, 2000).

[16] S. Sundaresan, Instabilities in fluidized beds, Annu. Rev. Fluid Mech. 35, 63 (2003).

[17] P. Duru, M. Nicolas, J. Hinch, and É. Guazzelli, Constitutive laws in liquid-fluidized beds, J. Fluid Mech. 452, 371 (2002).

[18] K. Anderson, S. Sundaresan, and R. Jackson, Instabilities and the formation of bubbles in fluidized beds, J. Fluid Mech. 303, 327 (1995).

[19] B. J. Glasser, I. G. Kevrekidis, and S. Sundaresan, One- and two-dimensional travelling wave solutions in gas-fluidized beds, J. Fluid Mech. 306, 183 (1996).

[20] J. Oshitani, T. Sasaki, T. Tsuji, K. Higashida, and D. Y. C. Chan, Anomalous Sinking of Spheres due to Local Fluidization of Apparently Fixed Powder Beds, Phys. Rev. Lett. 116, 068001 (2016).

[21] D. Kunii and O. Levenspiel, Fluidization Engineering (Butterworth-Heinemann, Boston, 1991).

[22] S. Chang, A gently aerated bed of glass beads sorts objects by density, Phys. Today 69 (4), 23 (2016).

[23] P. N. Rowe, B. A. Partridge, G. M. Ardran, and E. Lyall, Bubbles in fluidized beds, Nature 195, 278 (1962).

[24] R. F. Mudde, Time-resolved x-ray tomography of a fluidized bed, Powder Technol. 199, 55 (2010).

[25] B. Du, L. S. Fan, F. Wei, and W. Warsito, Gas and solids mixing in a turbulent fluidized bed, AIChE J. 48, 1896 (2002).

[26] E. Fukushima, Nuclear magnetic resonance as a tool to study flow, Annu. Rev. Fluid Mech. 31, 95 (1999).

[27] A. Penn, T. Tsuji, D. O. Brunner, C. M. Boyce, K. P. Pruessmann, and C. R. Muller, Real-time probing of granular dynamics with magnetic resonance, Sci. Adv. 3, e1701879 (2017).

[28] A. Penn, C. M. Boyce, T. Kovar, T. Tsuji, K. P. Pruessmann, and C. R. Muller, Real-time magnetic resonance imaging of bubble behavior and particle velocity in fluidized beds, Ind. Eng. Chem. Res. 57, 9674 (2018).

[29] C. M. Boyce, A. Penn, A. Padash, M. Lehnert, K. P. Pruessmann, and C. R. Muller, Anomalous collapse of interacting bubbles in a fluidized bed: A magnetic resonance imaging study, Phys. Rev. Fluids 4, 034303 (2019). 
[30] C. M. Boyce, A. Penn, K. P. Pruessmann, and C. R. Muller, Magnetic resonance imaging of gas solid fluidization with liquid bridging, AIChE J. 64, 2958 (2018).

[31] A. Penn, C. M. Boyce, N. Conzelmann, G. Bezinge, K. P. Pruessmann, and C. R. Muller, Real-time magnetic resonance imaging of fluidized beds with internals, Chem. Eng. Sci. 198, 117 (2019).

[32] A. Penn, Real-time magnetic resonance imaging of granular dynamics, Ph.D. thesis, ETH Zurich, 2018, https://doi.org/10.3929/ethz-b-000283101.

[33] Y. Tsuji, T. Kawaguchi, and T. Tanaka, Discrete particle simulation of 2-dimensional fluidized-bed, Powder Technol. 77, 79 (1993).

[34] T. Tsuji, K. Higashida, Y. Okuyama, and T. Tanaka, Fictitious particle method: A numerical model for flows including dense solids with large size difference, AIChE J. 60, 1606 (2014).

[35] D. Kolomenskiy and K. Schneider, A Fourier spectral method for the Navier-Stokes equations with volume penalization for moving solid obstacles, J. Comput. Phys. 228, 5687 (2009).

[36] K. Schneider, Numerical simulation of the transient flow behaviour in chemical reactors using a penalisation method, Comput. Fluids 34, 1223 (2005).

[37] S. Ergun, Fluid flow through packed columns, Chem. Eng. Prog. 48, 89 (1952).

[38] C. Y. Wen and Y. H. Yu, Mechanics of fluidization, Chem Eng. Prog. Symp. Ser. 62, 100 (1966).

[39] K. Iwashita and M. Oda, Rolling resistance at contacts in simulation of shear band development by DEM, J. Eng. Mech. 124, 285 (1998).

[40] M. Kobayakawa, S. Miyai, T. Tsuji, and T. Tanaka, Local dilation and compaction of granular materials induced by plate drag, Phys. Rev. E 98, 052907 (2018).

[41] T. Kawaguchi, T. Tanaka, and Y. Tsuji, Numerical simulation of fluidized bed using the discrete element method: The case of spouting bed, Trans. Jpn. Soc. Mech. Eng., B 58, 2119 (1992).

[42] S. Fazekas, J. Török, and J. Kertész, Critical packing in granular shear bands, Phys. Rev. E 75, 011302 (2007).

[43] G. Y. Onoda and E. G. Liniger, Random Loose Packings of Uniform Spheres and the Dilatancy Onset, Phys. Rev. Lett. 64, 2727 (1990).

[44] See Supplemental Material at http://link.aps.org/supplemental/10.1103/PhysRevFluids.6.064305 for (Supplemental Video 1) MR images of sphere sinking at $U_{0} / U_{\mathrm{mf}}=0.95$; (Supplemental Video 2) wiggling motion of sphere $\left(\rho_{\text {sphere }} / \rho_{\text {bulk }}=1.00, U_{0} / U_{\mathrm{mf}}=0.95\right)$; (Supplemental Video 3$)$ fluidizationsensitive MR imaging of slow and deep sinking $\left(\rho_{\text {sphere }} / \rho_{\text {bulk }}=1.00, U_{0} / U_{\mathrm{mf}}=0.95\right)$; (Supplemental Video 4) numerical results of sphere sinking at $U_{0} / U_{\mathrm{mf}}=0.95$; (Supplemental Video 5) bubble formation and detachment $\left(\rho_{\text {sphere }} / \rho_{\text {bulk }}=0.65\right.$ and 1.20, $\left.U_{0} / U_{\text {mf }}=0.95\right)$; (Supplemental Video 6) local void fraction change from initial state $\left[\Delta \varepsilon_{\text {local }}(t)=\varepsilon_{\text {local }}(t)-\varepsilon_{\text {local }}(t=0), \rho_{\text {sphere }} / \rho_{\text {bulk }}=1.2, U_{0} / U_{\text {mf }}=0.95\right]$.

[45] M. Suzuki and T. Oshima, Verification of a model for estimating the void fraction in a three-component randomly packed bed, Powder Technol. 43, 147 (1985).

[46] D. Lohse, R. Rauhé, R. Bergmann, and D. van der Meer, Creating a dry variety of quicksand, Nature 432, 689 (2004).

[47] H. C. Brinkman, A calculation of the viscous force exerted by a flowing fluid on a dense swarm of particles, Appl. Sci. Res. A1, 27 (1949).

[48] C. K. W. Tam, The drag on a cloud of spherical particles in low Reynolds number flow, J. Fluid Mech. 38, 537 (1969).

[49] T. H. Nguyen and J. R. Grace, Forces on objects immersed in fluidized-beds, Powder Technol. 19, 255 (1978).

[50] N. Gravish, P. B. Umbanhowar, and D. I. Goldman, Force and Flow Transition in Plowed Granular Media, Phys. Rev. Lett. 105, 128301 (2010). 